\title{
Vibroacoustic Analysis of a Rectangular Enclosure Bounded by a Flexible Panel with Clamped Boundary Condition
}

\author{
Yuan Wang, Jianrun Zhang, and Vanquynh Le \\ College of Mechanical Engineering, Southeast University, Nanjing 211189, China \\ Correspondence should be addressed to Jianrun Zhang; zhangjr@seu.edu.cn
}

Received 15 November 2012; Accepted 13 April 2013; Published 9 March 2014

Academic Editor: Reza Jazar

Copyright (C) 2014 Yuan Wang et al. This is an open access article distributed under the Creative Commons Attribution License, which permits unrestricted use, distribution, and reproduction in any medium, provided the original work is properly cited.

\begin{abstract}
A lot of research on the properties of a panel-enclosure coupled system which consists of an enclosure with a simply supported flexible wall was conducted. However, an enclosure with clamped flexible walls is commonly encountered. The properties of a panel-enclosure system, with one clamped flexible wall, are different from those of a simply supported one, and there are only a few studies on it. Thus, this paper is dedicated to the study of the effect of structural-acoustic coupling on the properties of a panel-enclosure system, which consists of an enclosure with a clamped panel. The resonance frequencies and the decay times of the coupled system are obtained using the classical modal coupling theory. The effects of enclosure and its flexible boundary on the resonance frequencies and the modal decay times of the coupled system are then analyzed. It is shown that, when panel thickness is changed, coupling strength is determined by the difference between the resonance frequencies of the panel and the enclosure modes. However, for the variation of enclosure depth, the factor which determines the coupling strength between panel and enclosure modes is the enclosure depth or the difference between their resonance frequencies.
\end{abstract}

\section{Introduction}

The interaction between a sound field in an enclosure and its flexible boundary is a critical problem, and a good understanding of it is particularly important to the control of sound field in an enclosure. Considerable effort has been devoted to the study of this classical model for many years. The works of Dowell $[1,2]$ represent some of early research into modeling of vibrations of panel backed by an enclosure. In the works of Pan of and Bies [3], the effect of acousticstructural coupling on the sound field in an enclosure, whose flexible wall is simply supported along its edges, was studied using the classical modal coupling method. However, the influence of enclosure depth and absorptive walls of enclosure on the coupling degree was not considered in this work. Kim and Brennan [4] proposed an impedance-mobility approach for the analysis of panel-enclosure system, and an enclosure with a simply supported wall was used to validate the proposed approach by experiment. In the efforts of Pan et al. [5] and Xin et al. [6,7], the weighted residual method was used to calculate the response of a panel-enclosure coupled system. Based on the analysis of acoustic-structural coupling, the forced response of panel-enclosure coupled system with different kinds of excitation [8-11] and active noise control [12-15] were studied. In the effort of Balachandran et al. [15], modal coupling method was used to study the active noise control of panel-enclosure coupled system, in which the flexible panel was clamped along its edges. However, the free vibration characteristics of a panel-enclosure coupled system were not analyzed.

The flexible wall of an enclosure with clamped boundary is commonly encountered, and the acoustic-structural coupling in panel-enclosure system which consists of an enclosure with a clamped flexible wall is different from that of a simply supported one. Thus, the boundary condition of panel has significant influence on the coupling with enclosure sound field. The panel with different boundary conditions has attracted many attentions using different methods. RayleighRitz approach was used to analyze the vibration and acoustic radiation of a baffled panel with arbitrary boundary conditions [16, 17], and a baffled and unbaffled panel in light and heavy fluid with arbitrary conditions [18]. In the efforts of 


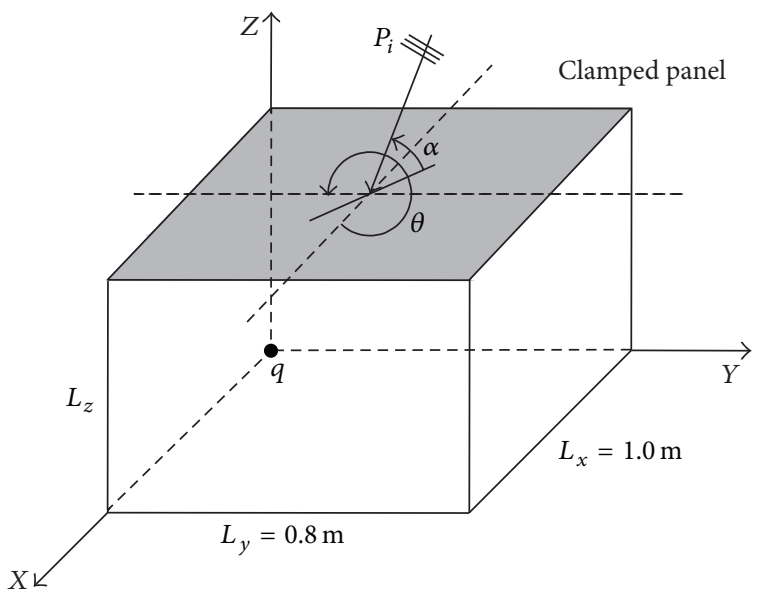

FIgURE 1: Panel-enclosure geometry and coordinate system.

Sung and Jan [19] and Arenas [20, 21], the transverse vibration response and sound radiation by a clamped panel were obtained using the virtual work principle. Compared with the Rayleigh-Ritz approach, virtual work principle provides an easier way to calculate the surface response of panel. In the current work, the mode shape function proposed by Sung and Arenas is used to analyze the characteristics of panelenclosure coupled system which consists of an enclosure with one clamped flexible wall.

In this paper, the vibroacoustic analysis of a rectangular enclosure, which is bounded by a clamped flexible wall, is conducted using the modal coupling method. Acoustical modes are used to describe the coupled system behavior. Firstly, the free vibration characteristics of panel-enclosure system are solved based on the modal parameters of uncoupled panel and enclosure, and the characteristics of coupled system which reflect the intrinsic properties are obtained. Secondly, the features of coupled system, which include resonance frequency and modal decay time, are analyzed with different variables, including panel thickness, panel internal damping, and enclosure depth. The energy transfer, resonance frequencies, and decay times of acoustical modes are distinguished from others when the panel mode and enclosure mode are well coupled. This work is an extension of the work carried by Pan and Bies [3] and Sung and Jan [19], where the effect of fluid-structural coupling on the sound field in the enclosure is studied with a simply supported flexible wall using the modal coupling method, and the clamped boundary panel with new mode shape function is used to analyze the vibration response, respectively.

\section{Analytical Model}

A panel-enclosure coupled system is shown in Figure 1. The enclosure has five small absorptive walls and one flexible wall, and the dimensions of enclosure are $L_{x}, L_{y}$, and $L_{z}$. The panel, which is clamped along its edges at $z=L_{z}$, has the dimensions of $L_{x}, L_{y} . P_{i n}$ is the amplitude of plane wave $P_{i}$, and the $\alpha, \theta$ are, respectively, the elevation and azimuth angles with respect to the panel center. $q$ is volume velocity of source in the enclosure, and it is located at origin of coordinate $(x, y, z)=(0,0,0) \mathrm{m}$. The sound pressure $p$ in the enclosure is described by an inhomogeneous wave equation:

$$
\nabla^{2} p-\frac{1}{c_{0}^{2}} \frac{\partial^{2} p}{\partial t^{2}}=-\rho_{0} \frac{\partial q}{\partial t}
$$

where $c_{0}, \rho_{0}$ are the speed of sound and air density, respectively. At the five absorptive walls inside the enclosure and the flexible enclosure interface, the boundary conditions take the following form:

$$
\begin{aligned}
& \frac{\partial p}{\partial \mathbf{n}}=-j \frac{\omega \rho_{0}}{Z_{A}} p \quad \text { at absorbent walls } \\
& \left.\frac{\partial p}{\partial z}\right|_{z=L_{z}}=-\rho_{0} \frac{\partial^{2} w}{\partial t^{2}} \quad \text { at flexible wall, }
\end{aligned}
$$

where $\mathbf{n}$ indicates the normal direction of the boundary surfaces. $Z_{A}$ is the specific acoustic impedance on the absorptive walls inside the enclosure. $w(x, y, z)$ is the normal displacement of flexible panel.

The governing equation of flexible vibration of thin isotropic panel due to the internal pressure $p$ and plane wave $p_{\text {in }}$ on the outside of the panel is

$$
\frac{\rho h \partial^{2} w}{\partial t^{2}}+D \nabla^{4} w=p-p_{i n}
$$

where $D=E h^{3} / 12\left(1-\mu^{2}\right)$ and $\rho, E, \mu, h$ are the density, Young's modulus, Poisson's ration, and thickness of the flexible panel, respectively. In this analysis, the panel radiation is neglected, and the positive direction for displacement of clamped panel is towards outside of the enclosure.

In order to use the modal shape expansion, the sound pressure $p$ inside enclosure, the flexible panel velocity $v=$ $j \omega w$, the sound pressure $p_{i n}$ on the outside of the panel, and the monopole acoustic source $q$ inside the enclosure can be expanded by mode shape functions:

$$
\begin{aligned}
& p(\mathbf{r})=\left[\phi_{N}\right]^{T}\left[p_{N}\right]=\boldsymbol{\Phi}^{\mathrm{T}} \mathbf{P}, \\
& v(\boldsymbol{\sigma})=\left[s_{M}\right]^{T}\left[v_{M}\right]^{T}=\mathbf{S}^{\mathrm{T}} \mathbf{V},
\end{aligned}
$$




$$
\begin{gathered}
p_{i n}(\boldsymbol{\sigma})=\left[s_{M}\right]^{T}\left[P_{i n, M}\right]^{T}=\mathbf{S}^{\mathbf{T}} \mathbf{P}_{i n}, \\
q(\mathbf{r})=\left[\phi_{N}\right]^{T}\left[Q_{N}\right]=\boldsymbol{\Phi}^{T} \mathbf{Q} .
\end{gathered}
$$

In these equations, $p_{N}, v_{M}, P_{i n, M}, Q_{N}$ are modal amplitude of sound pressure inside enclosure, flexible panel vibration velocity, the sound pressure on the outside of the clamped panel surface, and the acoustic source inside enclosure, respectively. $\phi_{N}, s_{M}$ are modal shape functions of rigidly walls enclosure and uncoupled clamped panel, respectively. Here, they are the base functions for the analysis of enclosure with modally reactive flexible panel and locally reactive walls.

The mode shape function $\phi_{N}$ of rigid wall enclosure is given by

$\phi_{N}(\mathbf{r})=\phi_{N}(x, y, z)=\cos \left(\frac{l \pi x}{L_{x}}\right) \cos \left(\frac{m \pi y}{L_{y}}\right) \cos \left(\frac{n \pi z}{L_{z}}\right)$.

Here, $l, m, n$ are the mode indices of the $N$ th enclosure mode.

The mode shape function $S_{M}$ of uncoupled clamped panel is given by Sung and Jan [19], and it has been validated by experiment:

$$
\begin{gathered}
s_{M}(\boldsymbol{\sigma})=s_{M}(x, y)=\psi_{u}(x) \psi_{v}(y), \\
\psi_{u}(x)=\gamma\left(\frac{\lambda_{u} x}{L_{x}}\right)-\frac{\gamma\left(\lambda_{u}\right)}{H\left(\lambda_{u}\right)} H\left(\frac{\lambda_{u} x}{L_{x}}\right), \\
\psi_{v}(y)=\gamma\left(\frac{\lambda_{v} y}{L_{y}}\right)-\frac{\gamma\left(\lambda_{v}\right)}{H\left(\lambda_{v}\right)} H\left(\frac{\lambda_{v} y}{L_{y}}\right),
\end{gathered}
$$

where $\gamma(s)=\cosh (s)-\cos (s), H(s)=\sinh (s)-\sin (s)$.

$\lambda_{u}, \lambda_{v}$ are determined by (10), and $u, v$ are mode indices of clamped panel modes:

$$
\cosh (\lambda) \cos (\lambda)-1=0 .
$$

Considering the acoustic boundary condition in (2) and using the Green function technique, the complex acoustic pressure $p$ in the enclosure can be expressed as

$$
p=j \omega \rho_{0} \int_{A_{f}} v G_{A} d s+j \omega \rho_{0} \int_{V_{0}} q G_{A} d V+j \frac{\omega}{c_{0}} \int_{A_{L}} \beta G_{A} p d s
$$

Here, $A_{f}, A_{l}, V_{0}$ are the surface of flexible panel, the absorbent wall surface inside enclosure, and the volume of enclosure, respectively. $\beta=\rho_{0} c_{0} / Z_{A}$ is the specific acoustic admittance ratio on the absorbent wall surface. $G_{A}$ is the sound field Green's function:

$$
G_{A}\left(\mathbf{r}, \mathbf{r}_{0}\right)=\sum_{N} \frac{\phi_{N}(\mathbf{r}) \phi_{N}\left(\mathbf{r}_{0}\right)}{V_{0} \Lambda_{N}\left(k^{2}-k_{a N}^{2}\right)}
$$

$$
\Lambda_{N}=\frac{1}{V_{0}} \int_{V_{0}} \phi_{N}^{2}(\mathbf{r}) d r
$$

Similarly, the flexible clamped panel vibration velocity can be expressed as

$$
v=j \frac{\omega}{\rho h} \int_{A_{f}}\left(p-p_{i n}\right) G_{p} d s
$$

$G_{p}$ is panel Green's function, which can be expressed as

$$
\begin{aligned}
G_{p}\left(\boldsymbol{\sigma}, \boldsymbol{\sigma}_{0}\right) & =-\sum_{M} \frac{s_{M}(\boldsymbol{\sigma}) s_{M}\left(\boldsymbol{\sigma}_{0}\right)}{A_{f} \Lambda_{M}\left(\omega^{2}-\omega_{p M}^{2}\right)}, \\
\Lambda_{M} & =\frac{1}{A_{f}} \int_{A_{f}} s_{M}^{2}(\boldsymbol{\sigma}) d \sigma .
\end{aligned}
$$
obtain

Substituting (4), (5), (6), and (7) into (11) and (13), we can

$$
(\lambda \mathbf{I}-\mathbf{A}) \mathbf{X}=\mathbf{Y}
$$

Equation (15) can be expressed as

$$
\left(\lambda \mathbf{I}-\left(\begin{array}{cc}
\mathbf{A}_{11} & \mathbf{A}_{12} \\
\mathbf{A}_{21} & \mathbf{A}_{22}
\end{array}\right)\right)\left(\begin{array}{c}
\mathbf{P} \\
\mathbf{V} \\
\lambda \mathbf{P} \\
\lambda \mathbf{V}
\end{array}\right)=\lambda\left(\begin{array}{c}
0 \\
0 \\
\mathbf{E}_{\mathbf{Q}} \\
\mathbf{E}_{\mathbf{F}}
\end{array}\right)
$$

where $\mathbf{I}$ is unit matrix, $\lambda=-j k . \mathbf{A}_{11}$ and $\mathbf{A}_{12}$ are $(N+M) \times$ $(N+M)$ zero and unit matrices, respectively:

$$
\begin{gathered}
\mathbf{E}_{\mathbf{Q}}=-c_{0} \rho_{0}\left(\begin{array}{llll}
Q_{1} & Q_{2} & \cdots & Q_{N}
\end{array}\right)^{T}, \\
\mathbf{E}_{\mathbf{F}}=\frac{1}{\rho h c_{0}}\left(\begin{array}{llll}
P_{i n, 1} & P_{i n, 2} & \cdots & P_{i n, M}
\end{array}\right)^{T},
\end{gathered}
$$

$$
\mathbf{A}_{21}=-\left[\begin{array}{cccccc}
k_{a 1}^{2} & & & & & \\
& \ddots & & 0 & & \\
& & k_{a N}^{2} & & & \\
& & & k_{p 1}^{2} & & \\
& 0 & & & \ddots & \\
& & & & & k_{p M}^{2}
\end{array}\right] \text {, }
$$




$$
\mathbf{A}_{22}=-\left[\begin{array}{c}
\eta_{a 1} \\
0 \\
-\frac{A_{f} B_{1,1}}{c_{0} M_{1}^{P}} \\
\vdots \\
A_{f} \int_{A_{f}} s_{M}(\boldsymbol{\sigma}) \phi_{N}(\mathbf{r}) d s \\
-\frac{4.4 \pi}{c_{0} M_{M}^{P}}
\end{array}\right.
$$

where $k_{a N}=\omega_{a N} / c_{0}, k_{p M}=\omega_{p M} / c_{0}, k_{a N}, \omega_{a N}$ are the wavenumber and the resonance angle frequency of the $N$ th enclosure mode, respectively. $k_{p M}$ and $\omega_{p M}$ are, respectively, the wavenumber and the resonance angle frequency of the $M$ th panel mode. $M^{P}, M^{A}$ are the modal mass of panel and enclosure, respectively. $M_{M}^{P}=\rho h A_{f} \Lambda_{M}, M_{N}^{A}=\rho_{0} V_{0} \Lambda_{N}$. $A_{f}, V_{0}$ are the area of flexible panel and volume of enclosure, respectively. $\eta_{p M}, \eta_{a N}$ are, respectively, the loss factor of $M$ th panel mode and $N$ th enclosure mode. Particularly, $\eta_{a N}$ is related to the integral of the specific acoustic admittance $\beta$. $T_{p M}, T_{a N}$ are, respectively, the $60 \mathrm{~dB}$ decay time of $M$ th panel mode and $N$ th enclosure mode.

\section{The Modal Properties of the Panel-Enclosure System}

3.1. Modal Coupling Coefficient. Modal coupling coefficient is the spatial matching degree between enclosure and panel modes on the interacting surface of enclosure sound field and flexible panel from (21). It is the integral of panel mode and enclosure mode on the surface of panel. Modal coupling coefficient only depends on the geometry shape and boundary condition of panel, the mode shape of enclosure acoustic field [3], and it has nothing to do with thickness, material properties, damping of the panel, and enclosure depth.

Like the panel-enclosure system which consists of an enclosure with a simply-supported flexible wall, the mode coupling coefficient between clamped panel and enclosure is nonzero, which must satisfy the following two conditions at the same time:

$$
\begin{gathered}
u+l=\text { odd number } \\
v+m=\text { odd number. }
\end{gathered}
$$

3.2. Transfer Factor. Coupling coefficient determines the matching degree between an enclosure mode and a panel mode on the interacting surface of enclosure sound field and panel, while the coupling extent between a panel mode and an enclosure mode is decided by transfer factor [3]. The transfer factor $F_{N, M}$ between the $N$ th enclosure acoustical mode and $M$ th panel mode is given by

$$
\begin{gathered}
F_{N, M}=\left\{1+\left[\frac{\left(\omega_{a N}-\omega_{p M}\right)}{2}\right]^{2}\left[\frac{1}{B(N, M)^{2}}\right]\right\}^{-1}, \\
B(N, M)=\left(\frac{\rho_{0} c_{0}^{2}}{\rho h L_{z} \Lambda_{N} \Lambda_{M}}\right)^{1 / 2} B_{N, M} .
\end{gathered}
$$

When the modal coupling coefficient between the $N$ th enclosure acoustical mode and $M$ th panel mode changes, the transfer factor $F_{N, M}$ has different results from (25) and (26). There are two different kinds of transfer factors: (1) as the modal coupling coefficient $B_{N, M}$ is equal to zero, the transfer factor $F_{N, M}$ must be equal to zero. In this situation, there is not energy transfer between the $N$ th enclosure acoustical mode and $M$ th panel mode. (2) When the modal coupling coefficient is nonzero, the transfer factor relates to many factors, such as the difference between resonance frequencies of uncoupled panel and enclosure modes and the enclosure depth. The larger the transfer factor between a panel mode and an enclosure mode is, the bigger the coupling strength between them is obtained. When the transfer factor $F_{N, M}$ is in the order of 1.0, the energy transfer between the Nth enclosure mode and $M$ th panel mode is important.

3.3. Resonance Frequency and Modal Decay Time of Coupled System. The sound pressure in the enclosure and the clamped panel vibration velocity are used to describe the response 
of coupled panel-enclosure system. Through the surface of flexible panel facing the inside of enclosure, the sound field in the enclosure is coupling with the vibration of flexible panel. There are two different kinds of acoustical modes in the panel-enclosure coupled system; one is an enclosurecontrolled acoustical mode whose most of energy is stored in the enclosure sound field and the other is a panel-controlled acoustical mode whose most of energy is stored as panel vibration energy [3].

If the external excitation does not exist in the coupled system, there will be $\mathbf{Y}=0$ in (15) and it becomes a $2(N+$ $M)$ dimensional system of equations. Corresponding to the eigenequation, there will be $2(N+M)$ eigenvalues $\lambda_{L}$ and $\lambda_{L}^{*}$, and $L=1,2 \cdots(N+M)$. The resonance frequency $f_{L}$ and the decay time $T_{L}$ of coupled system are $\operatorname{Im}\left(\lambda_{L}\right) c_{0} / 2 \pi$ and $6.91 / \operatorname{Re}\left(\lambda_{L}\right) c_{0}$, respectively. When the $\mathbf{Y} \neq 0$ in (15), the solution of the coefficient $\mathbf{X}$ is the modal amplitude of coupled system. Then, the panel vibration velocity and the sound pressure in the enclosure which describes the forced response of coupled system can be obtained from (4) and (5).

The time-averaged acoustic potential energy $E_{a N}$ in the enclosure and the time-averaged vibration kinetic energy $E_{p M}$ of the flexible panel are given by [14]

$$
\begin{gathered}
E_{a N}=\frac{1}{\left(4 \rho_{0} c_{0}^{2}\right) \int_{V_{0}}|p(\mathbf{r}, \omega)|^{2} d V}=\frac{V_{0} \mathbf{P}^{\mathbf{H}} \Lambda_{a N} \mathbf{P}}{\left(4 \rho_{0} c_{0}^{2}\right)}, \\
E_{p M}=\frac{\rho h}{4 \int_{A_{f}}|v(\boldsymbol{\sigma}, \omega)|^{2} d s}=\frac{\rho h A_{f} \mathbf{V}^{\mathbf{H}} \Lambda_{p M} \mathbf{V}}{4} .
\end{gathered}
$$

Here, $\Lambda_{a N}$ is a $N \times N$ diagonal matrix with each diagonal term consisting of $\Lambda_{N}$, and $\Lambda_{p M}$ is a $M \times M$ diagonal matrix with each diagonal term consisting of $\Lambda_{M}$.

\section{Results and Discussion}

To demonstrate the properties of the panel-enclosure coupled system which consists of an enclosure with a clamped flexible wall, the resonance frequencies and modal decay times of acoustical modes are investigated with different panel modal density, panel internal damping, and enclosure depth, respectively. The panel-enclosure coupled system, which consists of an enclosure with a clamped panel on top and five absorptive walls, is shown in Figure 1. The panel material properties are taken as follows: the material of clamped panel is aluminum, with density $\rho=2770 \mathrm{~kg} / \mathrm{m}^{3}$, Young's modulus $E=71 \mathrm{Gpa}$, and Poisson's ratio $\mu=0.33$.

\subsection{Effects of Different Panel Physical Parameters on Acoustical Modes}

4.1.1. Panel Modal Density. From (25), as the difference between resonance frequencies of uncoupled enclosure and panel modes is decreased, the transfer factors between them become larger when the modal coupling coefficient is nonzero. The resonance frequencies of rigid walls enclosure mode and uncoupled clamped panel mode [21] are given by

$$
\begin{aligned}
& f_{l, m, n}=\frac{c_{0}}{2}\left[\left(\frac{l}{L_{x}}\right)^{2}+\left(\frac{m}{L_{y}}\right)^{2}+\left(\frac{n}{L_{z}}\right)^{2}\right] \text {, } \\
& f_{u, v}=\frac{1}{2 \pi} \sqrt{\frac{D}{\rho}} \sqrt{\left(\frac{\lambda_{u}}{L_{x}}\right)^{4}+\left(\frac{\lambda_{v}}{L_{y}}\right)^{4}+2\left(\frac{\lambda_{u} \lambda_{v}}{L_{x} L_{y}}\right)^{2} \frac{\kappa_{u} \kappa_{v}}{\xi_{u} \xi_{v}}}, \\
& \kappa_{i}=\frac{1}{4}\left(1+D_{i}^{2}\right) \sinh \left(2 \lambda_{i}\right)-\frac{1}{2} D_{i} \cosh \left(2 \lambda_{i}\right) \\
& -\frac{1}{2}\left(1-D_{i}^{2}\right) \sin \left(\lambda_{i}\right) \cos \left(\lambda_{i}\right)-D_{i} \cos ^{2}\left(\lambda_{i}\right) \\
& -D_{i}^{2} \lambda_{i}+\frac{3}{2} D_{i} \\
& \xi_{i}=\frac{1}{4}\left(1+D_{i}^{2}\right) \sinh \left(2 \lambda_{i}\right) \\
& +\sinh \left(\lambda_{i}\right)\left[2 D_{i} \sin \left(\lambda_{i}\right)-\left(1-D_{i}^{2}\right) \cos \left(\lambda_{i}\right)\right] \\
& -\left(1+D_{i}^{2}\right) \sin \left(\lambda_{i}\right) \cosh \left(\lambda_{i}\right) \\
& +\frac{1}{2}\left(1-D_{i}^{2}\right) \sin \left(\lambda_{i}\right) \cos \left(\lambda_{i}\right)+\lambda_{i} \\
& -\frac{1}{2} D_{i}\left[1+\cosh \left(2 \lambda_{i}\right)\right]+D_{i} \cos ^{2}\left(\lambda_{i}\right), \\
& D_{i}=\frac{\gamma\left(\lambda_{i}\right)}{H\left(\lambda_{i}\right)}
\end{aligned}
$$

Similar to an enclosure with a simply supported flexible wall, the modal density of uncoupled clamped panel and enclosure will affect energy transfer between them. The modal density of uncoupled clamped panel and enclosure sound field are given by $[3,10]$

$$
\begin{gathered}
n_{p}=\frac{\sqrt{3} A_{f}}{C_{L} h}, \\
n_{a}=\frac{4 \pi V_{0} f^{2}}{c_{0}^{3}}+\frac{\pi S f}{2 c_{0}^{2}}+\frac{L}{8 c_{0}} .
\end{gathered}
$$

Here $A_{f}, C_{L}$ are the area and longitudinal wave speed of clamped flexible wall, respectively. $f$ is the excitation frequency. $S, L$ are the total surface area and the total edge lengths inside the enclosure.

As mentioned above, the transfer factor determines the coupling strength between a panel mode and an enclosure mode. From (34) and (25), by adjusting the panel modal density (corresponding to panel thickness), the distribution of the resonance frequencies of panel is changed, which also leads to changes of the transfer factors between panel and enclosure modes. In Figure 2 transfer factors between 


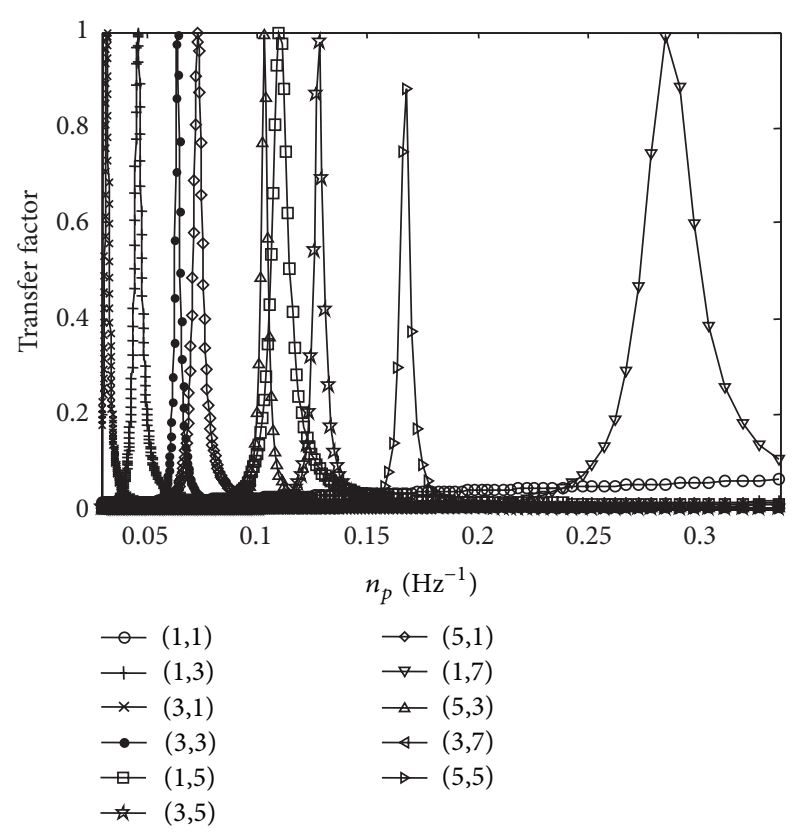

FIgURE 2: Transfer factors between the $(0,0,1)$ enclosure mode and panel modes as a function of panel modal density. $L_{Z}=0.6 \mathrm{~m}, T_{a N}=$ $15 \mathrm{~s}, T_{p M}=0.5 \mathrm{~s}$.

the $(0,0,1)$ enclosure mode and panel modes are plotted against panel modal density. When the panel modal density is low, only the $(3,1)$ panel mode participates in the coupling with the $(0,0,1)$ enclosure mode and satisfies the well-coupled condition. As the panel modal density is increased, more and more high-order panel modes, in which the coupling coefficients with the $(0,0,1)$ enclosure mode are not equal to zero, participate in coupling. At these points of transfer factors, when their values are in the order of 1.0, there is large energy transfer between enclosure and panel modes. Similar to the transfer factors between the $(0,0,1)$ enclosure mode and panel modes, the distribution of transfer factors between other enclosure modes and panel modes are that only few panel modes participate in the coupling with enclosure modes in the low panel modal density region and more panel modes participate in the coupling with enclosure mode for high panel modal density region.

As the coupling extent between enclosure and panel modes changes with the variation of panel modal density, the resonance frequency of acoustical mode, which is one important characteristic of it, is altered also. Figure 3(a) shows resonance frequencies of the first few enclosurecontrolled acoustical modes as a function of panel modal density. Forty panel modes and forty enclosure modes are used in this analysis. The decay times of all uncoupled enclosure and panel modes are $15 \mathrm{~s}$ and $0.5 \mathrm{~s}$, respectively. The depth of enclosure is $0.6 \mathrm{~m}$. As the panel modal density is increased, the resonance frequencies of enclosure-controlled modes jump to higher frequencies. Compared with the resonance frequencies of acoustical mode in the low panel modal density region, they become larger in the high panel modal density region. The reason is that many high-order panel modes participate in the coupling with enclosure modes. Similar to the panel-enclosure coupled system with a simply supported flexible panel, the energy transfer from the enclosure mode is distributed over many panel modes and no panel modes are well coupling with enclosure modes.

Figure 3(b) shows plot of the variation of decay times of the first few enclosure-controlled modes with panel modal density. When the panel modal density is small, the modal decay time is longer on the average. As the panel modal density is increased, the modal decay times show some minima. It is because of that only few panel modes satisfy the well-coupled condition with enclosure modes in the low panel modal density region. As the panel modal density is increased, more panel modes participate in the coupling with enclosure modes and decay times of enclosure-controlled modes become shorter. At the same time, there are no panel modes which are well coupled with enclosure mode, and the energy which is stored in the panel is equally distributed over many panel modes.

In order to describe the process of strong coupling between panel and enclosure modes when the panel modal density is altered, we analyze from three points of view of energy ratio between the panel vibration and sound field in the enclosure, resonance frequency, and modal decay time of coupled system, respectively. In this analysis, the plane wave $p_{i}$ with amplitude $1 \mathrm{~Pa}$ on the panel surface is used to drive the vibration of panel, the excitation frequencies are, respectively, the resonance frequencies of panel-controlled and enclosurecontrolled acoustical modes. The angles of elevation $\alpha$ and azimuth $\theta$ of plane wave are $90^{\circ}$ and $0^{\circ}$, respectively. The decay times of all uncoupled panel modes and enclosure modes are $15 \mathrm{~s}$ and $0.5 \mathrm{~s}$, respectively. As seen in Figure 2, the transfer factor between the $(3,1)$ panel mode and the $(0,0,1)$ enclosure mode is approximately equal to 1.0 in low panel modal density region and only these two modes satisfy wellcoupled condition, which means that the energy conversion between the sound field and the panel is almost entirely between these two modes.

The energy ratio between the panel vibration and sound field in the enclosure is shown in Figure 4(a), which contains panel-controlled and enclosure-controlled acoustical modes. The resonance frequencies of acoustical modes, uncoupled panel, and enclosure modes are shown in Figure 4(b) as a function of panel modal density. Figure 4(c) shows the variation of the decay time of acoustical modes with panel modal density. The effect of truncation numbers on the resonance frequencies of acoustical modes is shown in Figure 4(d). As the panel modal density tends to the point of transfer factor between the $(3,1)$ panel mode and the $(0,0,1)$ enclosure mode which is in the order of 1.0 , energy ratio, resonance frequencies, decay times of the $(3,1)$ panel-controlled mode, and the $(0,0,1)$ enclosure-controlled mode tend to be equal too.

Near the point of panel modal density where the maximum interaction strength between the $(3,1)$ panel mode and the $(0,0,1)$ enclosure mode, the energy which is stored in the panel vibration and enclosure sound field of two acoustical modes, including the $(0,0,1)$ enclosure-controlled mode and the $(3,1)$ panel-controlled mode, are approximately equal to 

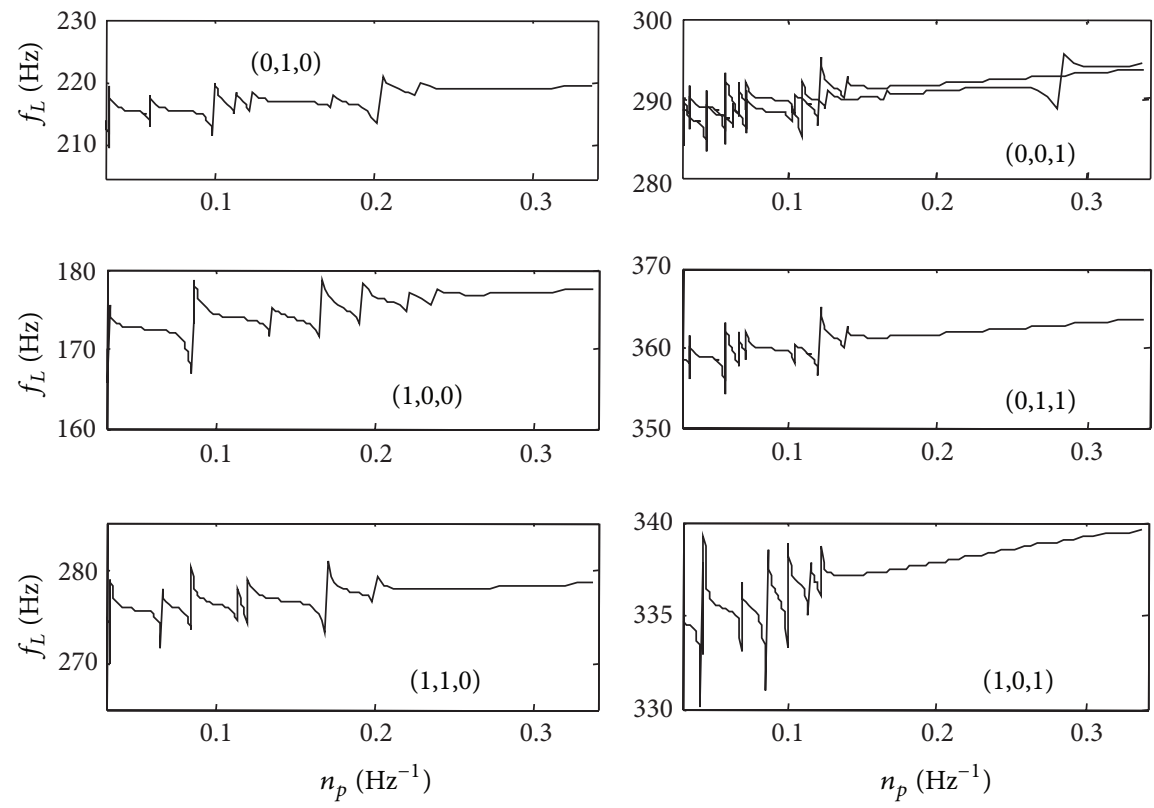

(a)
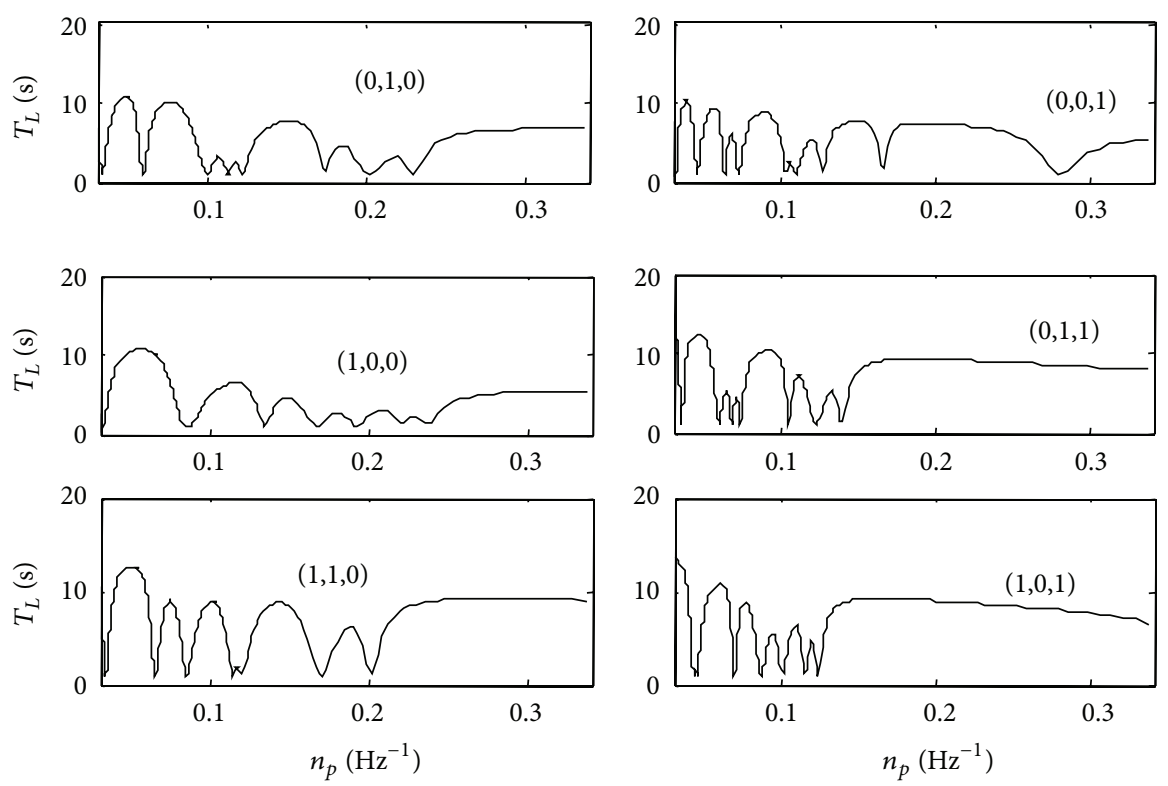

(b)

Figure 3: Resonance frequencies and decay times of the first few enclosure-controlled modes as a function of panel modal density: (a) resonance frequency, (b) decay time. $L_{Z}=0.6 \mathrm{~m}, T_{a N}=15 \mathrm{~s}, T_{p M}=0.5 \mathrm{~s}$.

each other, as shown in Figure 4(a). The further the distance from that point, the greater the difference between the energy ratio between the $(0,0,1)$ enclosure-controlled mode and the $(3,1)$ panel-controlled mode. The result also shows that energy ratio curve of the $(0,0,1)$ enclosure-controlled mode is a continuation of that of the $(3,1)$ panel-controlled mode as a function of panel modal density, and the energy ratio curve of the $(3,1)$ panel-controlled mode is a continuation of that of the $(0,0,1)$ enclosure-controlled mode.

It is shown in Figure 4(b) that, in the vicinity of the point of panel modal density where the transfer factor is approximately equal to 1.0 , the resonance frequencies of the $(0,0,1)$ enclosure-controlled mode and the $(3,1)$ panelcontrolled mode which deviate from uncoupled resonance frequencies are greater than others, respectively. The reason is that the strength of interaction between panel vibration and sound field in the enclosure becomes bigger than others. As the panel modal density is increased, the resonance frequency of the $(0,0,1)$ enclosure-controlled mode jumps from lower than the resonance frequency of the $(0,0,1)$ rigid walls enclosure mode to higher than it when it passes by the point of maximum energy transfer. Compared with the $(0,0,1)$ 


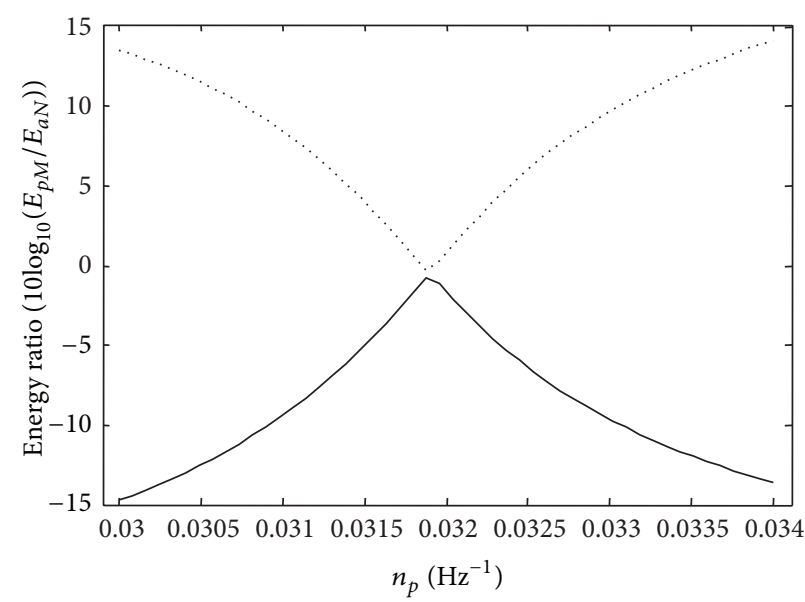

- $(0,0,1)$ enclosure-controlled mode

...... $(3,1)$ panel-controlled mode

(a)

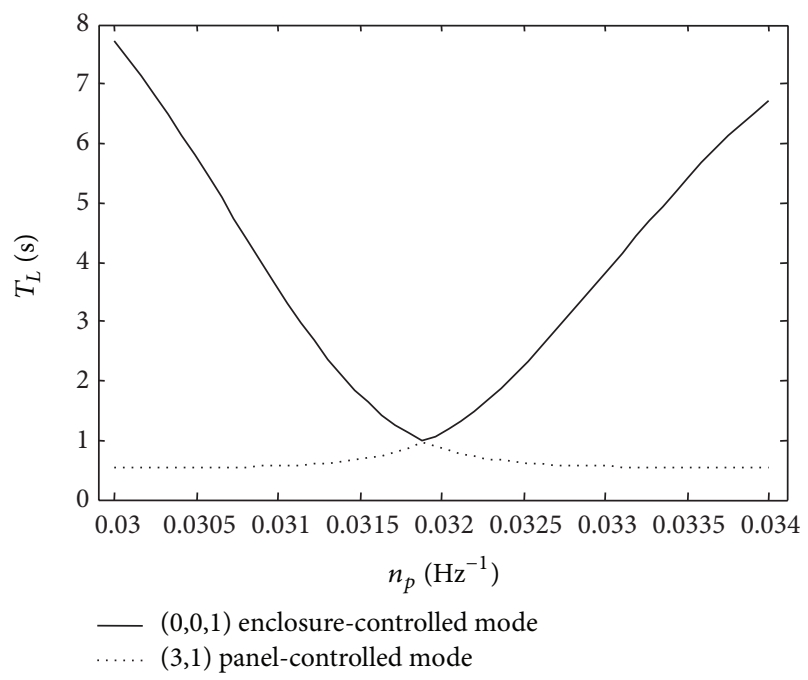

(c)

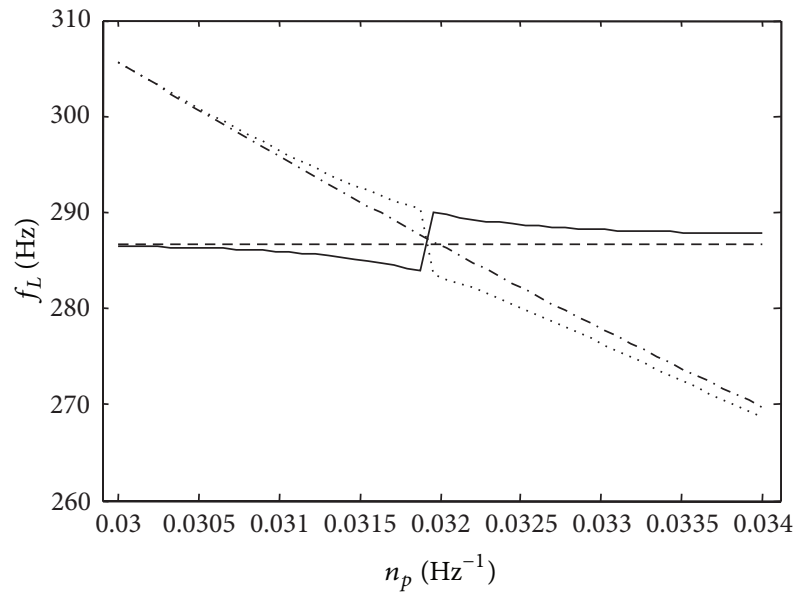

$(0,0,1)$ enclosure-controlled mode

..... $(3,1)$ panel-controlled mode

- . - $(0,0,1)$ uncoupled enclosure mode

- - - $(3,1)$ uncoupled panel mode

(b)

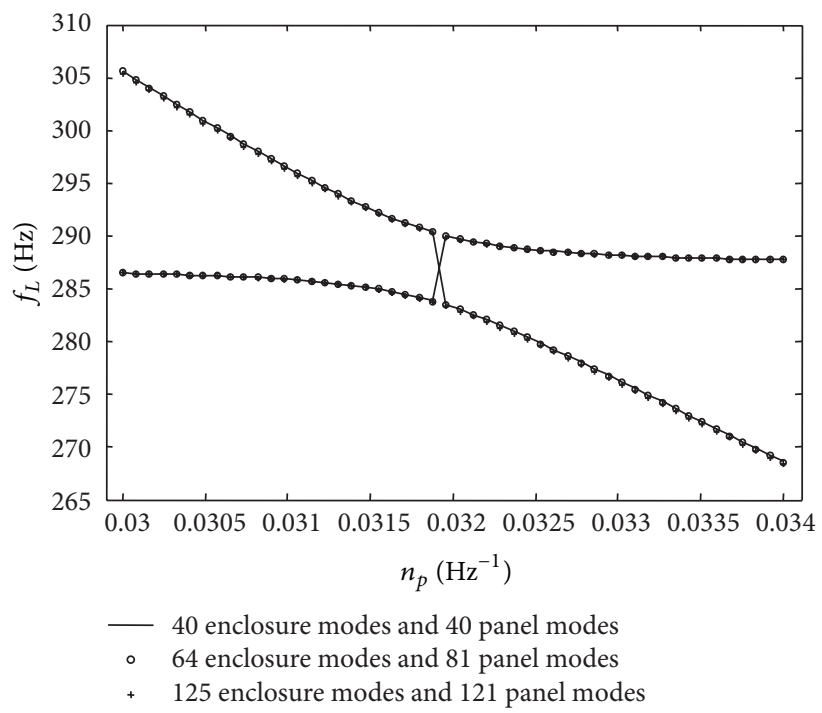

(d)

FIgURE 4: (a) Ratio between the sound field energy and panel vibration energy for two acoustical modes: $(0,0,1)$ enclosure-controlled mode and $(3,1)$ panel-controlled mode. (b) Resonance frequencies of two acoustical modes, the $(0,0,1)$ uncoupled enclosure mode, and the $(3,1)$ panel mode. (c) $60 \mathrm{~dB}$ modal decay time $T_{L}$. (d) Resonance frequencies of two acoustical modes with different truncation numbers. $L_{Z}=0.6 \mathrm{~m}$, $T_{p M}=0.5 \mathrm{~s}, T_{a N}=15 \mathrm{~s}$.

enclosure-controlled mode, it is opposite to the $(3,1)$ panelcontrolled mode. As the panel modal density is away from the point of maximum interaction strength, the resonance frequencies of the $(3,1)$ panel-controlled mode and the $(0,0,1)$ enclosure-controlled mode tend to resonance frequencies of uncoupled them, respectively.

It is shown in Figure 4(c) that, as the panel modal density is increased, decay time of the $(0,0,1)$ enclosure-controlled mode firstly reduces. When panel modal density arrives at the point of strong coupling, the minimum decay time is got. Then, decay time of the $(0,0,1)$ enclosure-controlled mode becomes longer as the panel modal density is increased. Compared with the $(0,0,1)$ enclosure-controlled mode, decay time curve of the $(3,1)$ panel-controlled mode is opposite as the panel modal density is increased. In addition, at the point of panel modal density where the decay times of the $(0,0,1)$ enclosure-controlled mode and the $(3,1)$ panel-controlled mode are approximately equal to each other, the energy transfer from the panel vibration to the enclosure acoustic field is relative maximum.

Due to the limited number of uncoupled panel and enclosure modes used in this analysis, some important modes may 


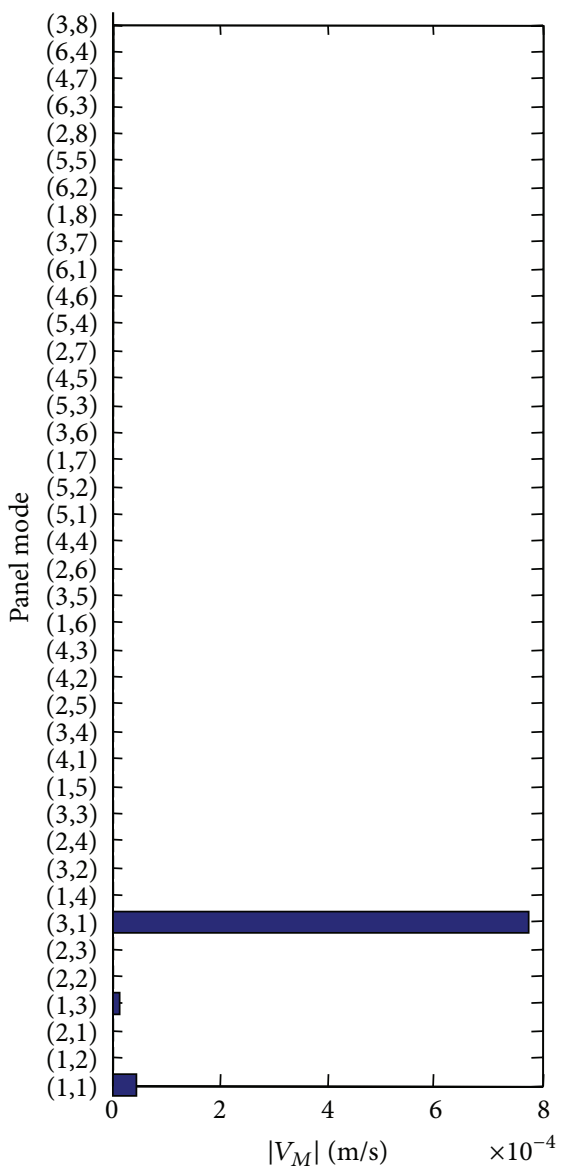

(a)

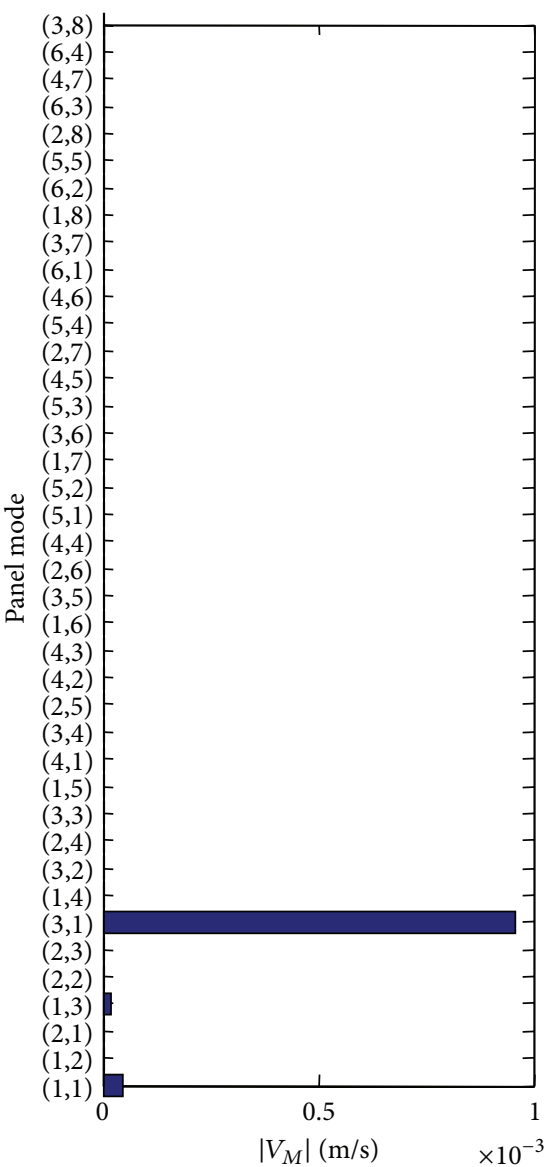

(b)

Figure 5: Modal amplitude of the panel vibration velocity: (a) the $(0,0,1)$ enclosure-controlled mode; (b) the $(3,1)$ panel-controlled mode. $h=0.00844 \mathrm{~m}, L_{Z}=0.6 \mathrm{~m}, T_{a N}=15 \mathrm{~s}, T_{p M}=0.5 \mathrm{~s}$.

be excluded, and truncation errors exist in the calculation of resonance frequencies and decay times of acoustical modes. Figure 4(d) shows the resonance frequencies of two acoustical modes using different truncated numbers. Compared with the solution for the resonance frequencies using the combination of 64 enclosure modes and 81 panel modes and of 125 enclosure modes and 121 panel modes, the solution for the resonance frequencies of the $(0,0,1)$ enclosure-controlled and the $(3,1)$ panel-controlled acoustical modes using 40 panel modes and 40 enclosure modes meets the requirement in this analysis, and the computation efficiency is also improved.

When the panel thickness is $0.0882 \mathrm{~m}$, the two subsystems modal amplitudes of panel vibration and enclosure acoustic field are shown in Figures 5 and 6, respectively. The panel vibration of coupled system is controlled by the $(3,1)$ panel mode in Figures 5(a) and 5(b). The sound field in the enclosure of coupled system is controlled by the $(0,0,1)$ enclosure mode in Figures 6(a) and 6(b). Combined with energy ratio shown in Figure 4(a), it is proved that the coupled system is named the $(3,1)$ panel-controlled acoustical mode and the $(0,0,1)$ enclosure-controlled acoustical mode, respectively.
4.1.2. Panel Internal Damping. The decay times of uncoupled clamped panel modes are used to describe the mechanical damping of the clamped panel in the analysis. The panel vibration energy is dissipated by its internal damping, and then the vibration level of panel will be reduced. The disturbing degree of the enclosure sound field due to the panel vibration becomes smaller, and the energy flow between the panel and enclosure becomes smaller too. The decay times and the resonance frequencies of enclosure-controlled modes are related to the decay times of uncoupled panel modes. Figures 7(a) and 7(b) show plot of the variation of the resonance frequencies and the decay times of the first few enclosure-controlled modes with the decay time of panel modes, respectively. In this analysis, the decay times of all rigid wall enclosure modes are $15 \mathrm{~s}$. The thickness of panel is $0.007 \mathrm{~m}$, and the depth of enclosure is $0.6 \mathrm{~m}$. The decay times of all uncoupled panel modes are equal.

In Figure 7(a), the resonance frequencies of the first few enclosure-controlled modes approach the uncoupled enclosure resonance frequency as the panel modal decay time is reduced (corresponding to the damping increased). The bigger the damping in the panel is, the more the energy is 


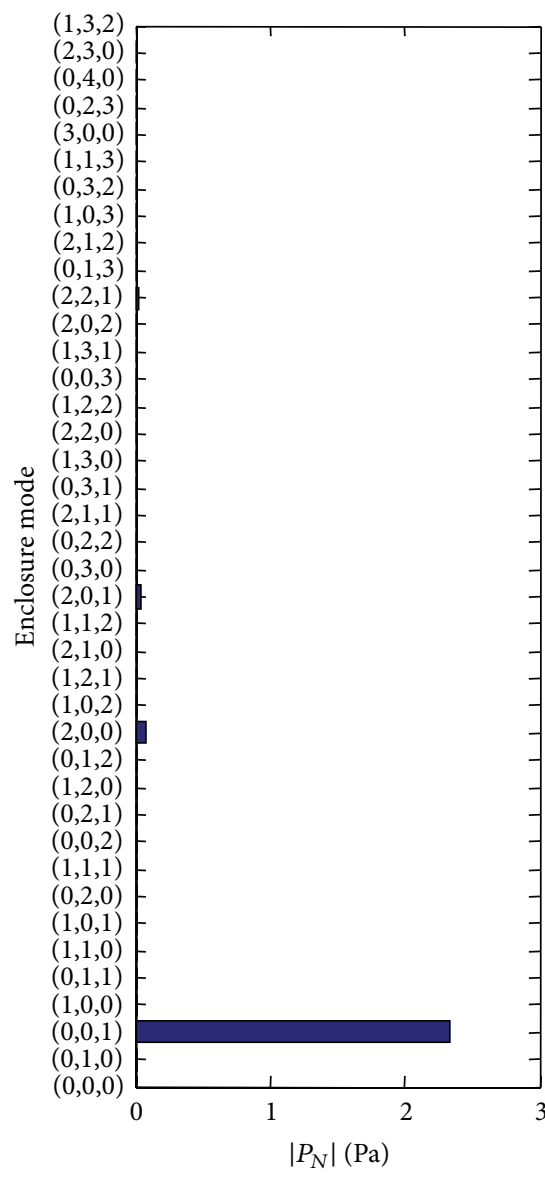

(a)

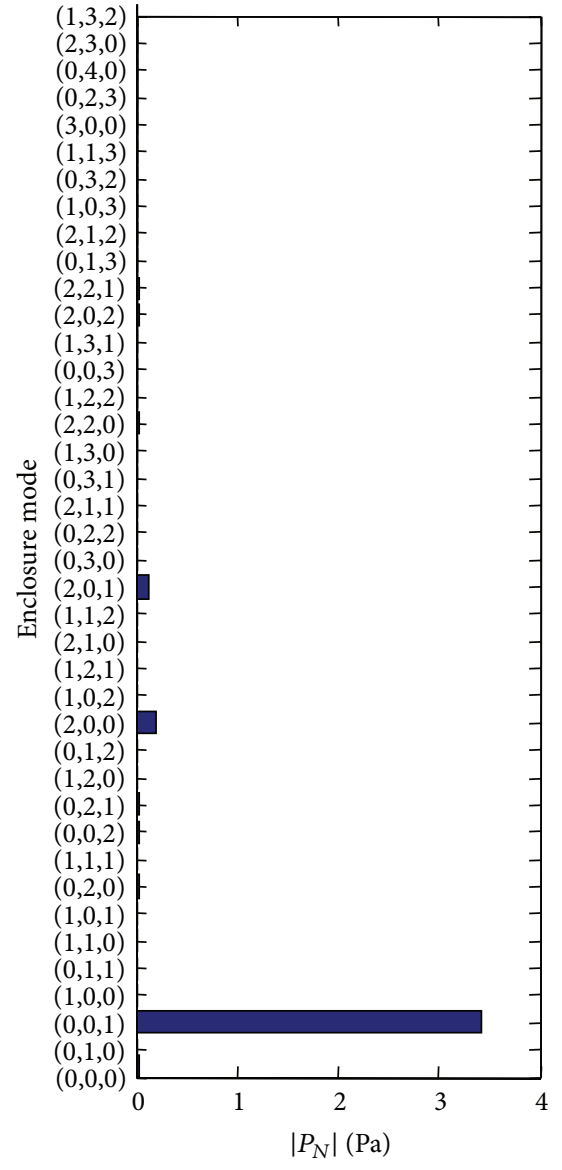

(b)

Figure 6: Modal amplitude of sound filed in the enclosure: (a) the $(0,0,1)$ enclosure-controlled mode; (b) the $(3,1)$ panel-controlled mode. $h=0.00844 \mathrm{~m}, L_{Z}=0.6 \mathrm{~m}, T_{a N}=15 \mathrm{~s}, T_{p M}=0.5 \mathrm{~s}$.

dissipated in the panel. As the damping of panel is increased, the flexible panel tends to rigid wall gradually, and the sound field in the enclosure will not be affected by the panel.

As the panel damping is increased, the decay times of the first few enclosure-controlled modes decrease and arrive at the minimum, and then they increase to the uncoupled enclosure mode, as shown in Figure 7(b).

4.2. Effects of Enclosure Depth on Acoustical Modes. Similar to the panel modal density, enclosure depth has significant effect on the panel-enclosure coupled system. Due to the modal density of enclosure sound field which relates to many factors from (35), such as excitation frequency $f$, enclosure volume $V_{0}$, total surface area of enclosure $S$, and total edge lengths inside enclosure $L$, the enclosure depth is used as a variable which affects the dimensions of enclosure in subsequent analysis. From (25), (26), and (29), when the coupling coefficient between enclosure and panel modes is not equal to zero, the reason for the variation of transfer factor between them with the change of enclosure depth can be divided into two different kinds: one is the change of difference between resonance frequencies of panel and enclosure modes when the enclosure modal index $n \neq 0$, and the other is the change of enclosure depth when the enclosure modal index $n=0$. The effect of enclosure depth on the resonance frequencies of panel-controlled acoustical mode was studied by Dowell et al. [2], but only resonance frequencies of the first two panel-controlled modes were studied.

As mentioned above, the coupling strength between panel and enclosure modes will be altered by adjusting enclosure depth when the modal coupling coefficient between them is nonzero. In Figures 8(a), 8(b), and 8(c), transfer factors between enclosure modes and panel modes $(1,1),(1,2)$, and $(3,1)$ are plotted against enclosure depth, respectively. Compared with the effect of panel modal density upon transfer factors, enclosure depth has smaller influence upon transfer factors in the zone of analysis.

In Figures 8(a) and 8(b), the transfer factors between enclosure modes and panel modes $(1,1)$ and $(1,2)$ are all less than 1.0, and it means that no enclosure modes satisfy the well-coupled condition with panel modes $(1,1)$ and $(1,2)$. But when the enclosure depth is shallow, the enclosure modes $(0,0,0)$ and $(0,1,0)$ have relative large coupling strength with clamped panel modes $(1,1)$ and $(1,2)$, respectively. As the enclosure depth is increased, the coupling strength between 

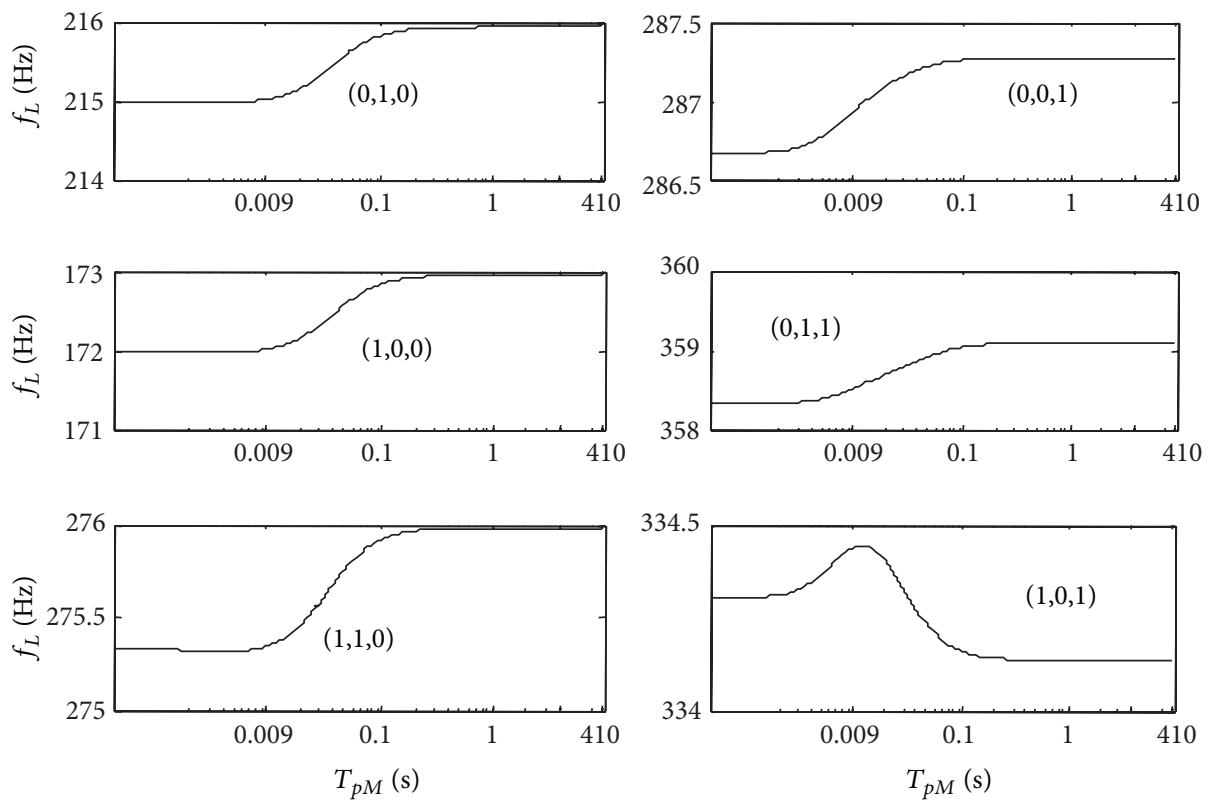

(a)
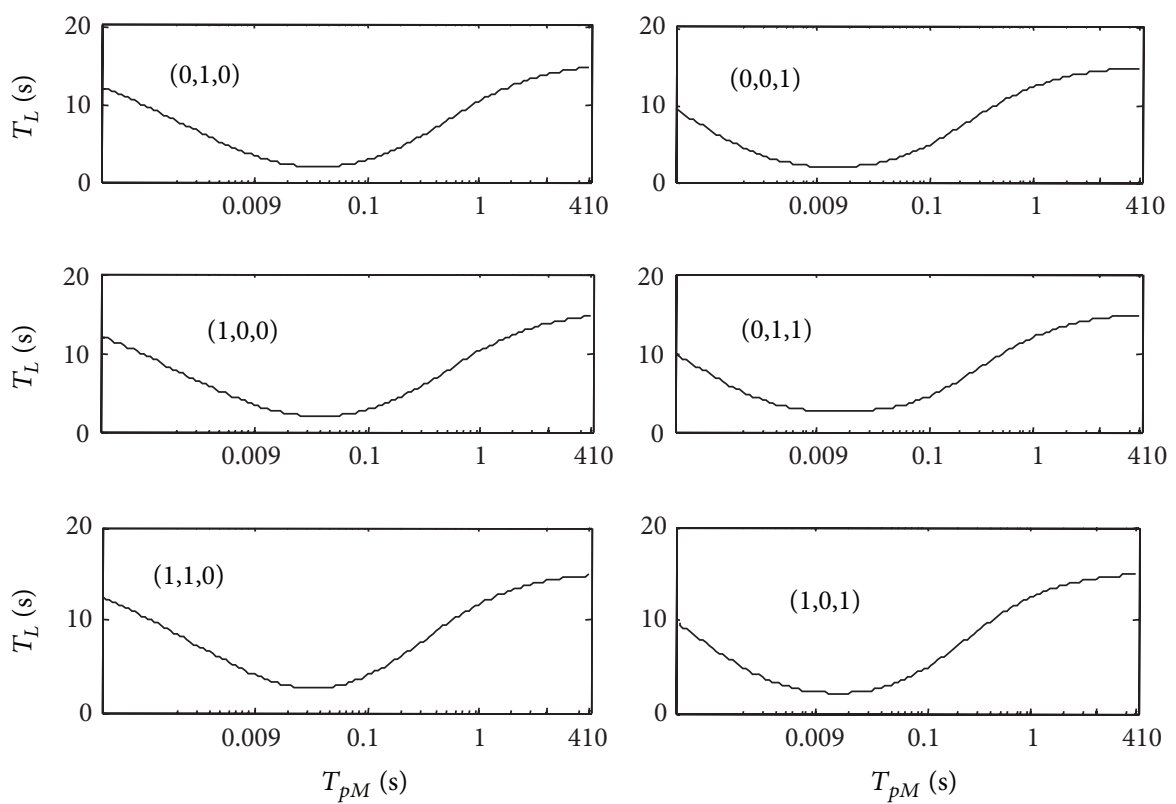

(b)

Figure 7: Resonance frequencies and decay times of the first few enclosure-controlled modes as a function of panel modal decay time: (a) resonance frequency, (b) decay time. $h=0.007 \mathrm{~m}, L_{Z}=0.6 \mathrm{~m}, T_{a N}=15 \mathrm{~s}$.

the $(0,0,0)$ enclosure mode and the $(1,1)$ panel mode decreases gradually and it is similar to transfer factor between the $(0,1,0)$ enclosure mode and the $(1,2)$ panel mode. The coupling degree between the $(0,0,1)$ enclosure mode and the $(1,1)$ panel mode is inverse when the enclosure depth is increased, and the influence of it upon transfer factor is less than enclosure mode $(0,0,0)$. The reason is that, when the mode index $n$ is equal to zero, the factor which determines the transfer factor between the enclosure and panel modes is for enclosure depth rather than for the difference between resonance frequencies of them from (25) and (26).

The transfer factor between the $(0,0,1)$ enclosure mode and the $(3,1)$ panel mode is in the order of 1.0 at the enclosure depth $0.7182 \mathrm{~m}$, as shown in Figure 8(c), and there will be large energy transfer between the $(0,0,1)$ enclosure mode and the $(3,1)$ panel mode. The reason is that the difference between resonance frequencies of enclosure and panel modes is in the order of 0 which is different from transfer factor between 


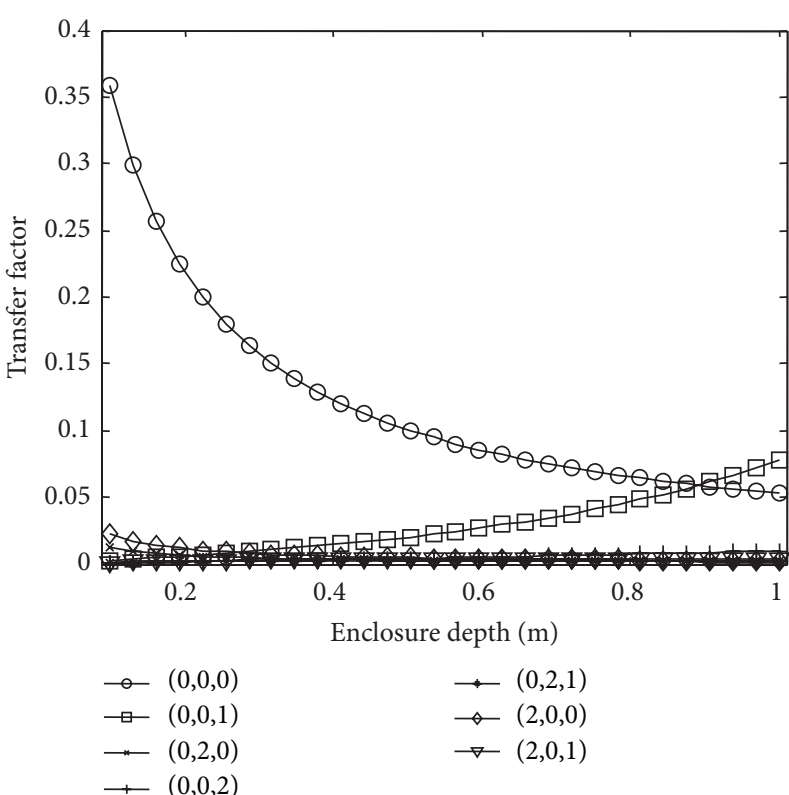

(a)

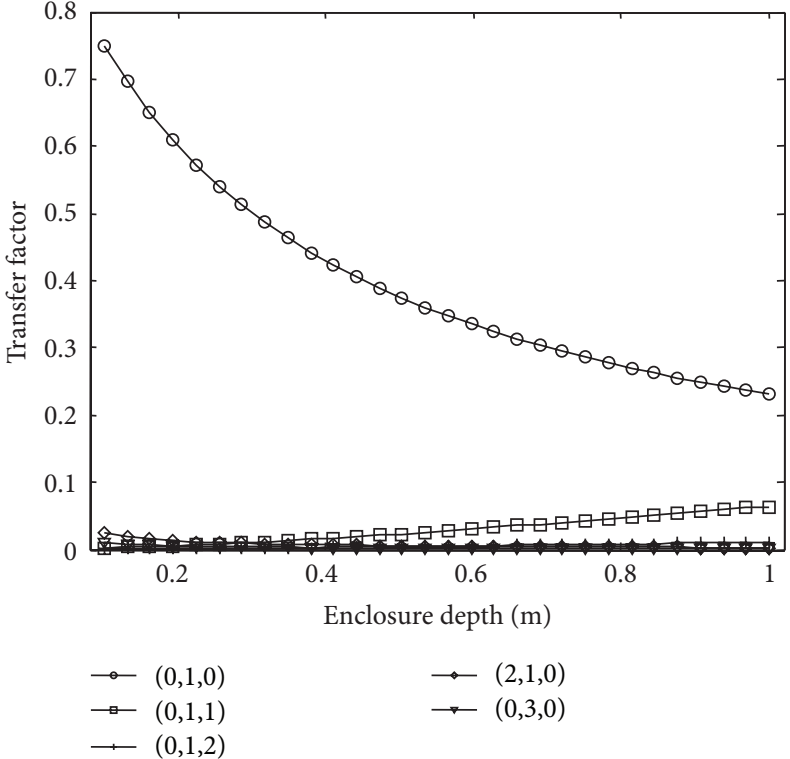

(b)

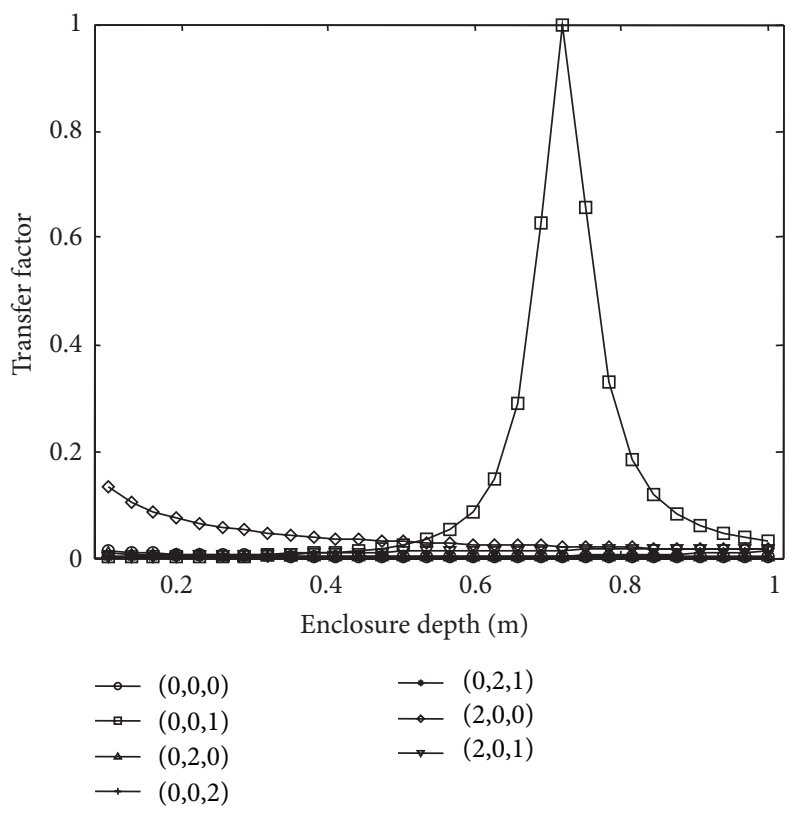

(c)

Figure 8: Transfer factors between enclosure modes and panel modes as a function of enclosure depth. (a) $(1,1)$ panel mode; (b) (1,2) panel mode; (c) $(3,1)$ panel mode; $h=0.007 \mathrm{~m}, T_{a N}=15 \mathrm{~s}, T_{p M}=0.5 \mathrm{~s}$.

the $(0,0,0)$ enclosure mode and the $(1,1)$ panel mode above. When the enclosure depth is shallow, the $(2,0,0)$ enclosure mode has small coupling strength with the $(3,1)$ panel mode, and the coupling strength is reduced with the increase in enclosure depth.

Figure 9(a) shows resonance frequencies of the first eight panel-controlled acoustical modes as a function of enclosure depth. As the enclosure depth is increased, the resonance frequencies of panel-controlled acoustical modes $(1,1),(1,2)$,
$(2,1)$, and $(2,2)$ approach to those of uncoupled ones. The reason is that transfer factors between panel modes above and enclosure modes are less than 1.0 and do not satisfy well-coupled condition between them in the frequency zone of analysis, and the variation of transfer factors between panel modes $(2,1),(2,2)$ and enclosure modes with enclosure depth is the same as panel modes $(1,1)$ and $(1,2)$, as shown in Figures 8(a) and 8(b). The resonance frequencies of panelcontrolled acoustical modes $(1,2),(2,1)$, and $(2,2)$ increase 

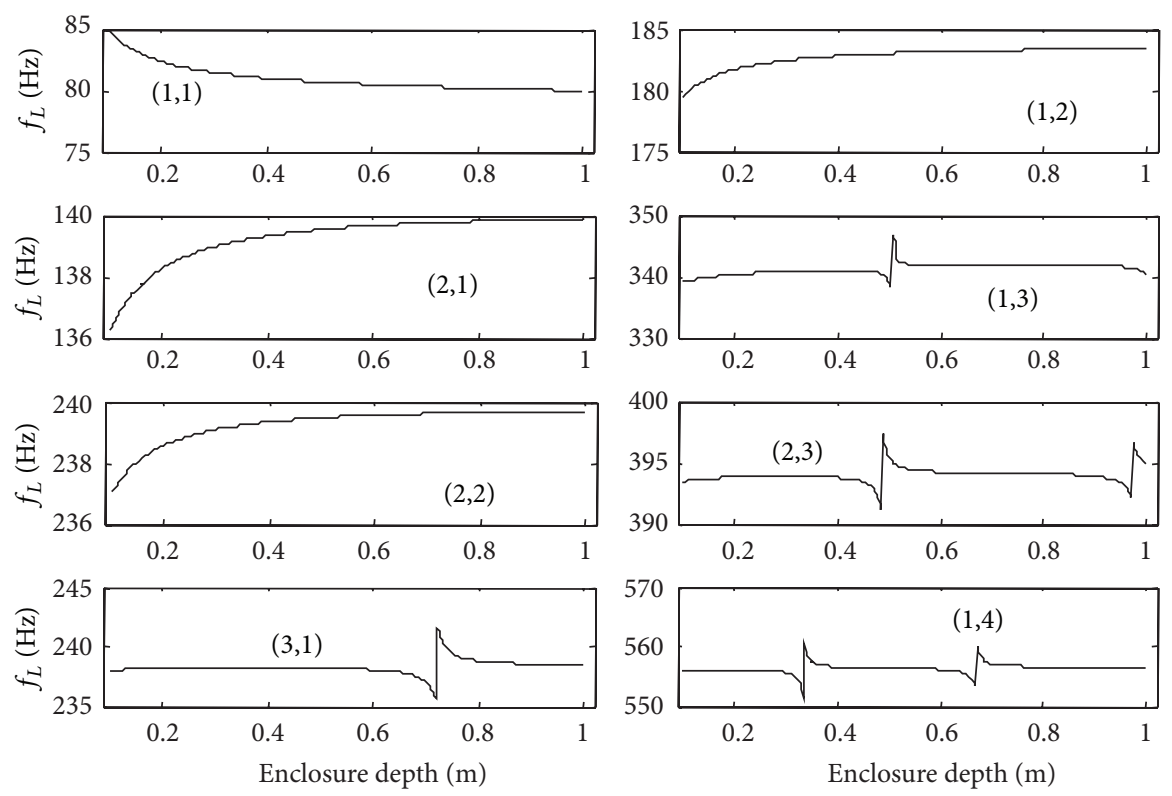

(a)
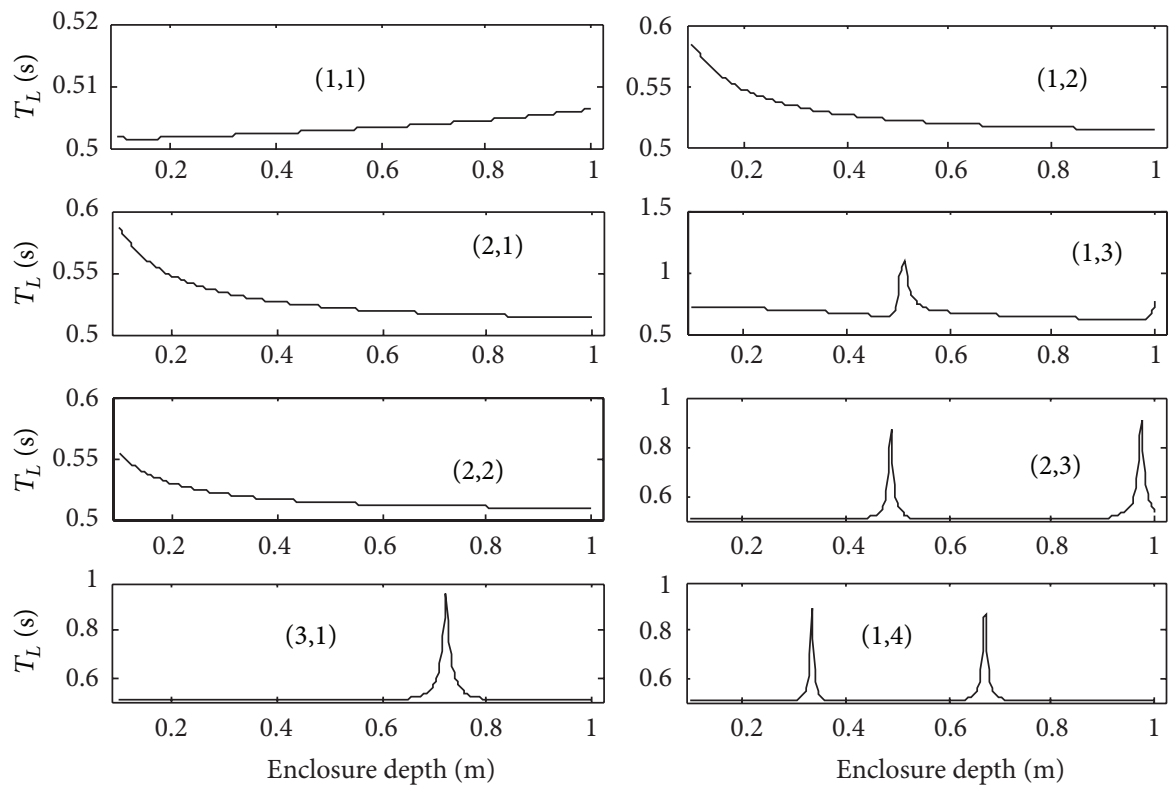

(b)

Figure 9: Resonance frequencies and decay times of the first few panel-controlled modes as a function of enclosure depth: (a) resonance frequency, (b) decay time. $h=0.007 \mathrm{~m}, T_{a N}=15 \mathrm{~s}, T_{p M}=0.5 \mathrm{~s}$.

gradually as the enclosure depth is increased. On the contrary, the resonance frequency of the $(1,1)$ panel-controlled mode is reduced. This is because of that the resonance frequencies of the $(1,1)$ uncoupled panel mode are larger than the $(0,0,0)$ rigid wall enclosure mode, while the resonance frequencies of panel modes $(1,2),(2,1),(2,2)$ is less than those of enclosure modes. The resonance frequencies of panel-controlled modes $(1,3),(2,3),(3,1)$, and $(1,4)$ appear jump phenomenon in the process of the change of enclosure depth, which corresponds to the point of enclosure depth, where the transfer factor between the $(3,1)$ panel mode and enclosure mode is in the order of 1.0. The variation of transfer factor between panel modes $(1,3),(2,3)$, and $(1,4)$ and enclosure modes is the same as the $(3,1)$ panel mode. Simultaneously, the interaction between panel and enclosure modes becomes stronger and the larger energy transfer between them is conducted.

In Figure 9(b) the decay times of the first eight panelcontrolled modes are plotted against enclosure depth. Corresponding to the points of enclosure depth where resonance frequencies of panel-controlled modes jump to higher frequencies in Figure 9(a), the decay times of them appear peaks. Meanwhile there will be large energy transfer between enclosure and panel modes. As the enclosure depth is increased, less enclosure modes participate in the coupling 


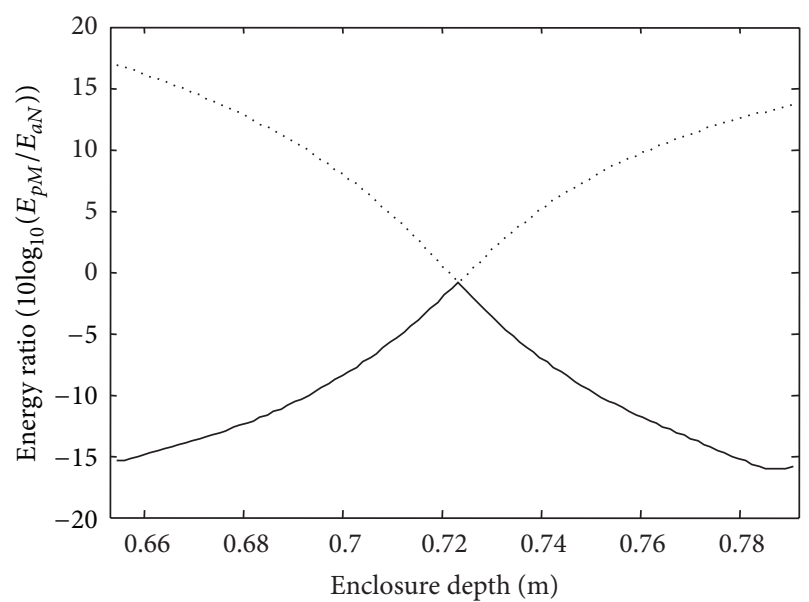

- $(0,0,1)$ enclosure-controlled mode $(3,1)$ panel-controlled mode

(a)

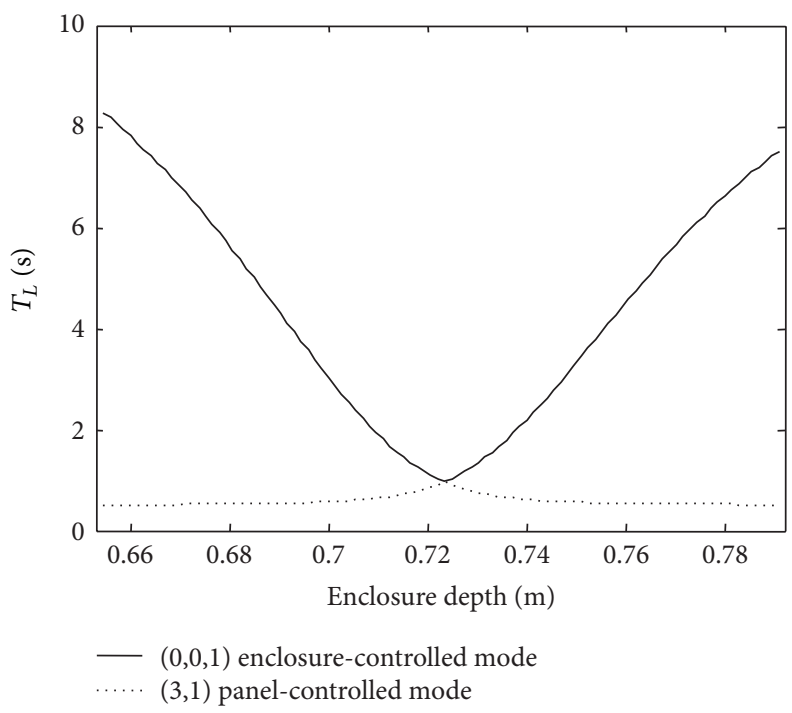

(c)

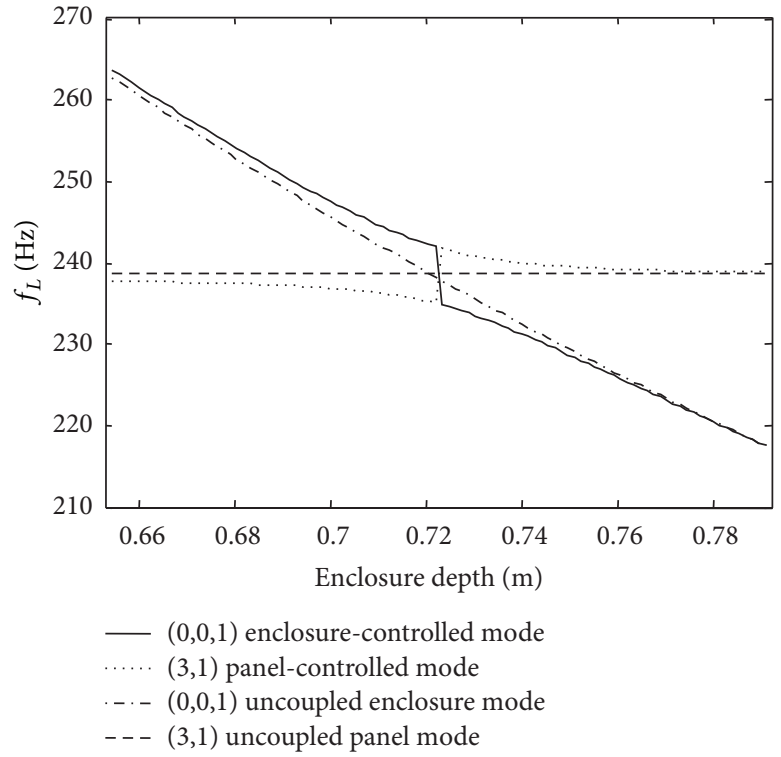

(b)

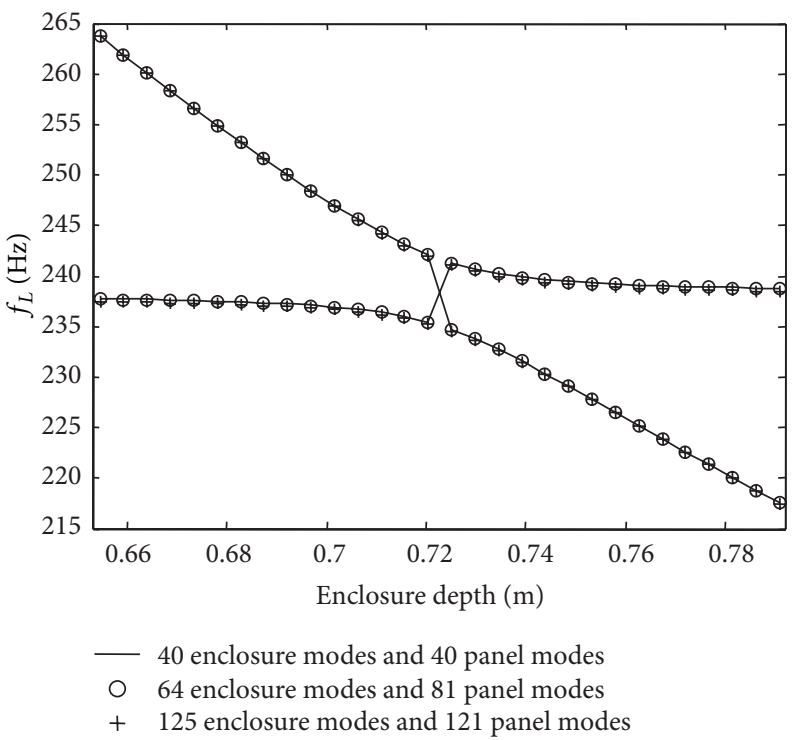

(d)

FIGURE 10: (a) Ratio between the panel vibration energy and sound field energy for two acoustical modes: $(0,0,1)$ enclosure-controlled mode and $(3,1)$ panel-controlled mode. (b) Resonance frequencies of two acoustical modes, the $(0,0,1)$ uncoupled enclosure mode, and the $(3,1)$ panel mode. (c) $60 \mathrm{~dB}$ modal decay time $T_{L}$. (d) Resonance frequencies of two acoustical modes with different truncation numbers. $h=0.007 \mathrm{~m}$, $T_{a N}=15 \mathrm{~s}, T_{p M}=0.5 \mathrm{~s}$.

with panel modes and the decay times of panel-controlled modes tend to those of uncoupled ones.

In order to describe the process of strong coupling between panel mode and enclosure mode when the enclosure depth is changed, we analyze the coupled system from three points of view of energy ratio between the panel vibration and sound field in the enclosure, the resonance frequencies, and modal decay times of coupled system. Similar to the analysis of forced response of the coupled system with different panel modal density, the plane wave $P_{i}$ with amplitude $1 \mathrm{~Pa}$ on the panel surface is also used to drive the vibration of panel, and the excitation frequencies are also, respectively, the resonance frequencies of panel-controlled and enclosurecontrolled acoustical modes. The elevation angle $\alpha$ and azimuth angle $\theta$ of plane wave are $90^{\circ}$ and $0^{\circ}$, respectively. The decay times of all uncoupled panel and enclosure modes are $15 \mathrm{~s}$ and $0.5 \mathrm{~s}$, respectively. As seen in Figure 8(c), the transfer factor between panel mode $(3,1)$ and enclosure mode $(0,0,1)$ is approximately equal to 1.0 at large enclosure depth, which means that the energy transfer between the sound field and the panel is almost entirely between these two modes.

The effect of enclosure depth on energy ratio between the sound field in the enclosure and panel vibration is shown in Figure 10(a), which contains a panel-controlled mode and 


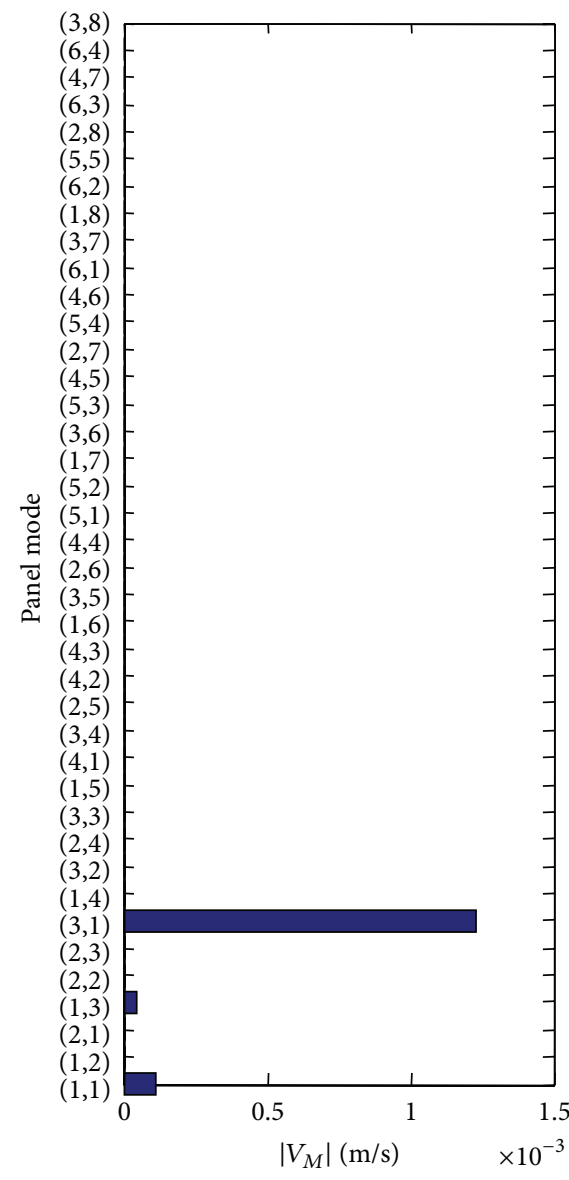

(a)

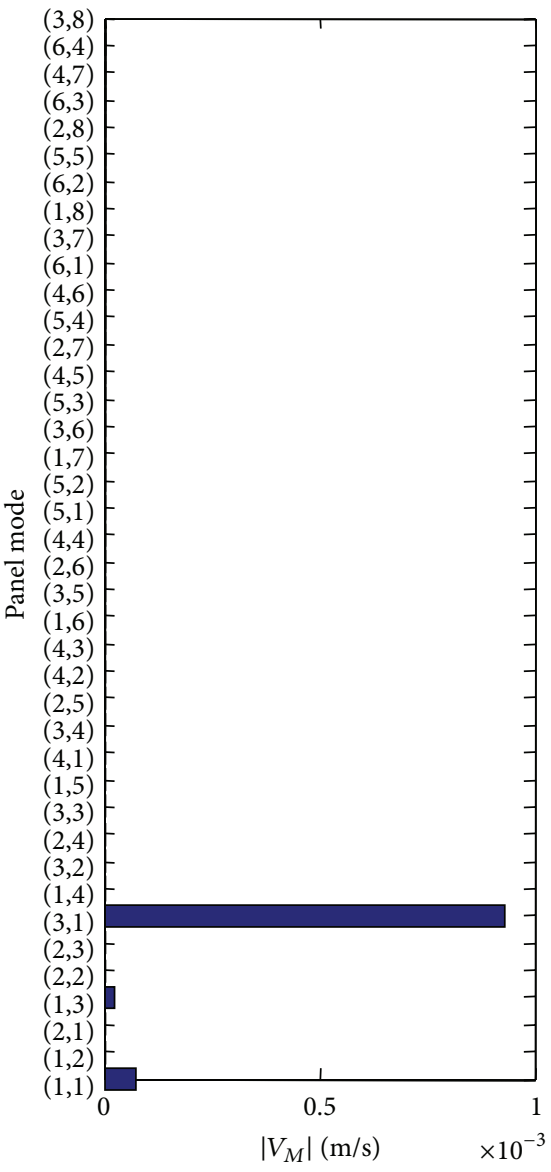

(b)

FIGURE 11: Modal amplitude of panel vibration velocity: (a) the $(0,0,1)$ enclosure-controlled mode; (b) the $(3,1)$ panel-controlled mode. $h=$ $0.007 \mathrm{~m}, L_{Z}=0.722 \mathrm{~m}, T_{a N}=15 \mathrm{~s}, T_{p M}=0.5 \mathrm{~s}$.

an enclosure-controlled mode. The resonance frequencies and the decay times of coupled system are plotted against enclosure depth in Figures 10(b) and 10(c), respectively. Figure 10(d) shows natural frequencies of two acoustical modes using three kinds of panel and enclosure modes numbers. In addition, the resonance frequencies of uncoupled panel and enclosure are included in Figure 10(b). As the enclosure depth tends to the point where transfer factor between the $(3,1)$ panel mode and the $(0,0,1)$ enclosure mode is in the order of 1.0, energy ratio, resonance frequencies, and decay times of the $(3,1)$ panel-controlled mode and the $(0,0,1)$ enclosure-controlled mode tend to be equal, respectively.

Similar to the effect of panel modal density on the energy ratio, near the point of the maximum interaction strength between the $(3,1)$ panel mode and the $(0,0,1)$ enclosure mode, the energy which is stored in each part (panel and enclosure) of the $(3,1)$ panel-controlled mode and of the $(0,0,1)$ enclosure-controlled mode approximately is equal, as shown in Figure 10(a). The further the distance from that point, the greater the difference between the energy ratio between the $(0,0,1)$ enclosure-controlled mode and the $(3,1)$ panel-controlled mode. And the energy ratio curves of the $(3,1)$ panel-controlled mode and the $(0,0,1)$ enclosurecontrolled mode are continuation of those of the $(0,0,1)$ enclosure-controlled mode one and the $(3,1)$ panel-controlled mode one as the enclosure depth is increased, respectively.

It is shown in Figure 10(b) that, in the vicinity of the point of enclosure depth where the transfer factor is approximately equal to 1.0 , the resonance frequencies of the $(0,0,1)$ enclosure-controlled mode and the $(3,1)$ panelcontrolled mode which deviate from uncoupled resonance frequencies are greater than others, respectively. The reason is that the strength of interaction between panel vibration and sound field in the enclosure becomes bigger than others. As the enclosure depth is away from the point of maximum interaction strength, the resonance frequencies of the $(3,1)$ panel-controlled mode and the $(0,0,1)$ enclosure-controlled mode tend to those of uncoupled ones, respectively.

In Figure 10(c), as the enclosure depth is increased, the decay time of the $(0,0,1)$ enclosure-controlled mode decreases gradually. When arriving at the point of strong coupling, the decay time becomes relative minimum. Then, the decay time of the $(0,0,1)$ enclosure-controlled mode become to increase as the enclosure depth is increased. Compared with the $(0,0,1)$ 


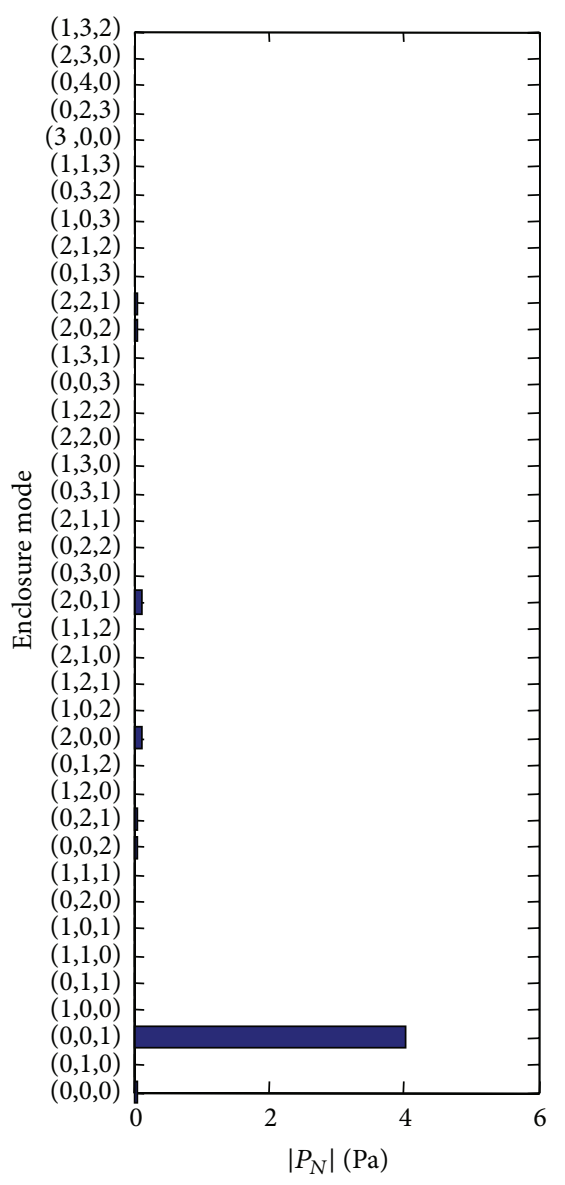

(a)

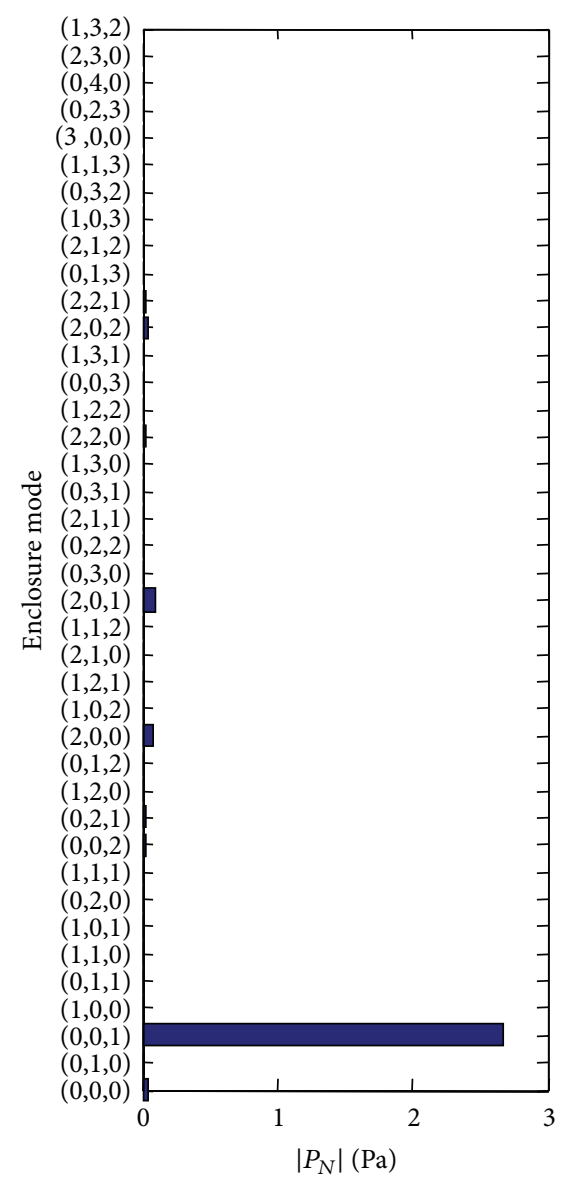

(b)

Figure 12: Modal amplitude of sound field in the enclosure: (a) the $(0,0,1)$ enclosure-controlled mode; (b) the $(3,1)$ panel-controlled mode. $h=0.007 \mathrm{~m}, L_{Z}=0.722 \mathrm{~m}, T_{a N}=15 \mathrm{~s}, T_{p M}=0.5 \mathrm{~s}$.

enclosure-controlled mode, the decay time curve of the $(3,1)$ panel-controlled mode is opposite as the enclosure depth is increased. In addition, at the point of panel modal density where the decay times of the $(0,0,1)$ enclosure-controlled mode and the $(3,1)$ panel-controlled mode tend to equal each other, the energy transfer between the $(0,0,1)$ enclosure mode and the $(3,1)$ panel mode is relative maximum for these two acoustical modes.

Similarly with the results shown in Figure 4(d), the truncation error of the solution for the resonance frequencies of $(0,0,1)$ enclosure-controlled and $(3,1)$ panel-controlled acoustical modes using 40 panel modes and 40 enclosure modes can be neglected, as shown in Figure 10(d).

When enclosure depth is $0.722 \mathrm{~m}$, modal amplitude of each part (panel and enclosure) of two acoustical modes is shown in Figures 11 and 12, respectively. Panel vibration of coupled system is controlled by the $(3,1)$ panel mode in Figures 11(a) and 11(b). Sound field in the enclosure of coupled system is controlled by the $(0,0,1)$ enclosure mode in Figures 12(a) and 12(b). Combined with the energy ratio shown in Figure 10(a), it is proved that the coupled system is named the $(3,1)$ panel-controlled mode and the $(0,0,1)$ enclosurecontrolled mode, respectively.

\section{Conclusions}

This paper presents a theoretical investigation into the vibroacoustic analysis of a rectangular enclosure with clamped flexible wall using the classical modal coupling method.

The coupling between clamped panel and enclosure modes is very selective, and it is the same as the coupling between enclosure and simply supported panel modes. The modal coupling coefficient determines the degree of match between panel and enclosure modes, and the coupling strength between panel and enclosure is determined by the transfer factor. When the panel modal density is changed, the coupling strength between panel and enclosure modes only depends on the difference between the resonance frequencies of them. But for the enclosure depth, the factors which determine the coupling strength between enclosure and panel modes can be divided into two different kinds: one is the difference between resonance frequencies of panel and enclosure modes when the enclosure modal index $n \neq 0$, and the other is the enclosure depth when the enclosure modal index $n=0$.

The transfer factor tends to 1.0 by adjusting the panel thickness or enclosure depth, and the interaction between 
the sound field in the enclosure and the panel vibration then becomes stronger gradually. In the vicinity of the maximum coupling point, the resonance frequencies of enclosure-controlled or panel-controlled modes appear jump phenomenon. Simultaneously, the resonance frequency and the decay time of acoustical mode which deviate from those of uncoupled ones are more than others, and the energy of interaction between panel vibration and sound field in enclosure becomes bigger than others.

The vibration of clamped panel which acts on the sound field in the enclosure can be changed by varying damping of the panel, and then the energy between the vibration panel and enclosure sound field is altered consequently.

\section{Conflict of Interests}

The authors declare that there is no conflict of interests regarding the publication of this paper.

\section{Acknowledgments}

This work is supported by the Science and Technology Support Plan of Jiangsu China (Grant no. BE2D1047) and combination of product, study, and investigation in the prospective Research Program of Jiangsu China (Grant no. BY2011151).

\section{References}

[1] E. H. Dowell and H. Voss, "The effect of a cavity on panel vibration," AIAA Journal, vol. 1, pp. 476-477, 1963.

[2] E. H. Dowell, G. F. Gorman III, and D. A. Smith, "Acoustoelasticity: general theory, acoustic natural modes and forced response to sinusoidal excitation, including comparisons with experiment," Journal of Sound and Vibration, vol. 52, no. 4, pp. 519-542, 1977.

[3] J. Pan and D. A. Bies, "The effect of fluid-structural coupling on sound waves in an enclosure-theoretical part," Journal of the Acoustical Society of America, vol. 87, no. 2, pp. 691-707, 1990.

[4] S. M. Kim and M. J. Brennan, "A compact matrix formulation using the impedance and mobility approach for the analysis of structural-acoustic systems," Journal of Sound and Vibration, vol. 223, no. 1, pp. 97-112, 1999.

[5] J. Pan, S. J. Elliott, and K.-H. Baek, "Analysis of low frequency acoustic response in a damped rectangular enclosure," Journal of Sound and Vibration, vol. 223, no. 4, pp. 543-566, 1999.

[6] F. X. Xin, T. J. Lu, and C. Q. Chen, "Vibroacoustic behavior of clamp mounted double-panel partition with enclosure air cavity," Journal of the Acoustical Society of America, vol. 124, no. 6, pp. 3604-3612, 2009.

[7] F. X. Xin and T. J. Lu, "Analytical and experimental investigation on transmission loss of clamped double panels: implication of boundary effects," Journal of the Acoustical Society of America, vol. 125, no. 3, pp. 1506-1517, 2009.

[8] J. Pan, "The forced response of an acoustic-structural coupled system," Journal of the Acoustical Society of America, vol. 91, no. 2, pp. 949-956, 1992.

[9] K. S. Sum and J. Pan, "An analytical model for bandlimited response of acoustic-structural coupled systems. I. Direct sound field excitation," Journal of the Acoustical Society of America, vol. 103, no. 2, pp. 911-923, 1998.

[10] K. S. Sum and J. Pan, "A study of the medium frequency response of sound field in a panel-cavity system," Journal of the Acoustical Society of America, vol. 103, no. 3, pp. 1510-1519, 1998.

[11] B. Venkatesham, M. Tiwari, and M. L. Munjal, "Analytical prediction of the breakout noise from a rectangular cavity with one compliant wall," Journal of the Acoustical Society of America, vol. 124, no. 5, pp. 2952-2962, 2008.

[12] J. Pan, C. H. Hansen, and D. A. Bies, "Active control of noise transmission through a panel into a cavity: I. Analytical Study," Journal of the Acoustical Society of America, vol. 87, no. 5, pp. 2098-2108, 1990.

[13] J. Pan and C. H. Hansen, "Active control of noise transmission through a panel into a cavity. III: effect of a actuator location," Journal of the Acoustical Society of America, vol. 90, no. 3, pp. 1493-1501, 1991.

[14] S.-M. Kim and M. J. Brennan, "Active control of harmonic sound transmission into an acoustic enclosure using both structural and acoustic actuators," Journal of the Acoustical Society of America, vol. 107, no. 5, pp. 2523-2534, 2000.

[15] B. Balachandran, A. Sampath, and J. Park, "Active control of interior noise in a three-dimensional enclosure," Smart Materials and Structures, vol. 5, no. 1, pp. 89-97, 1996.

[16] A. Berry, J.-L. Guyader, and J. Nicolas, "A general formulation for the sound radiation from rectangular, baffled plates with arbitrary boundary conditions," Journal of the Acoustical Society of America, vol. 88, no. 6, pp. 2792-2802, 1990.

[17] X. Zhang and W. L. Li, "A unified approach for predicting sound radiation from baffled rectangular plates with arbitrary boundary conditions," Journal of Sound and Vibration, vol. 329, no. 25, pp. 5307-5320, 2010.

[18] H. Nelisse, O. Beslin, and J. Nicolas, "A generalized approach for the acoustic radiation from a baffled or unbaffled plate with arbitrary boundary conditions, immersed in a light or heavy fluid," Journal of Sound and Vibration, vol. 211, no. 2, pp. 207225, 1998.

[19] C.-C. Sung and J. T. Jan, "The response of and sound power radiated by a clamped rectangular plate," Journal of Sound and Vibration, vol. 207, no. 3, pp. 301-317, 1997.

[20] J. P. Arenas, "On the vibration analysis of rectangular clamped plates using the virtual work principle," Journal of Sound and Vibration, vol. 266, no. 4, pp. 912-918, 2003.

[21] J. P. Arenas, Analysis of the acoustic radiation resistance matrix and its applications to vibro-acoustic problems [Ph.D. thesis], University of Auburn, 2001. 

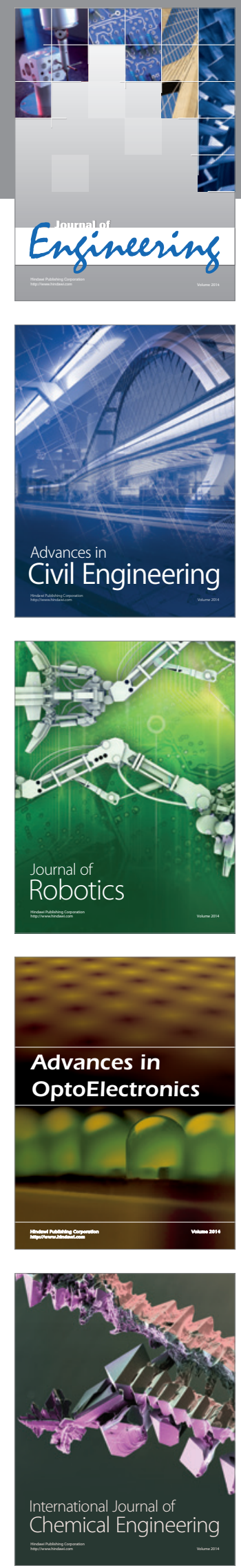

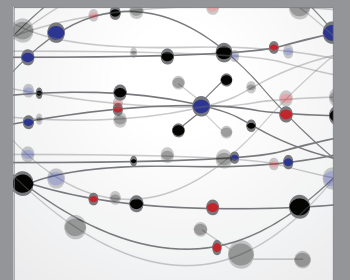

The Scientific World Journal
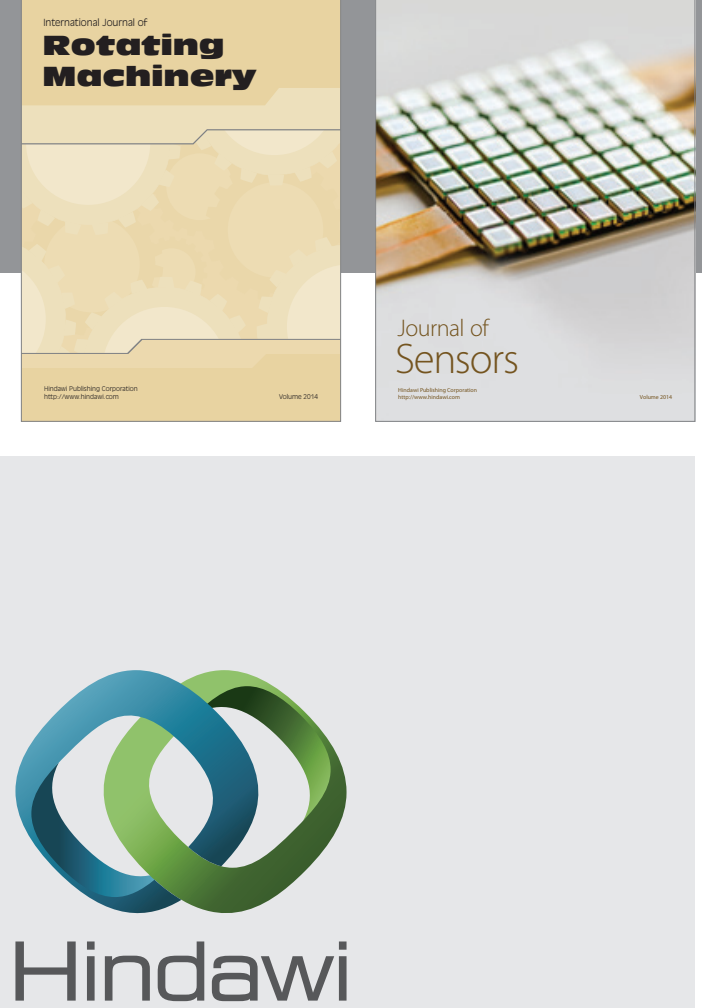

Submit your manuscripts at http://www.hindawi.com
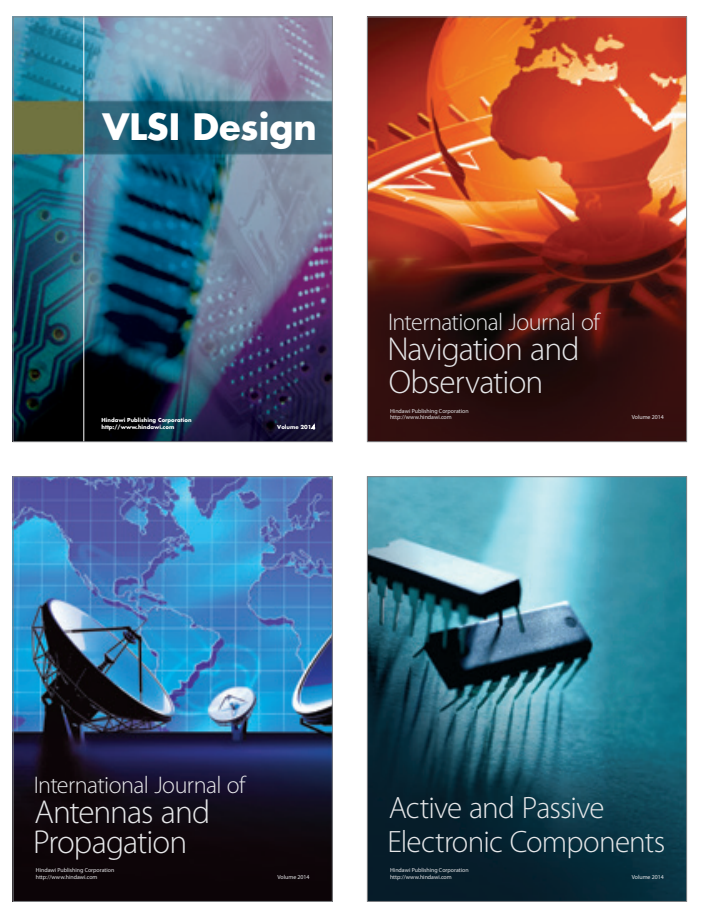
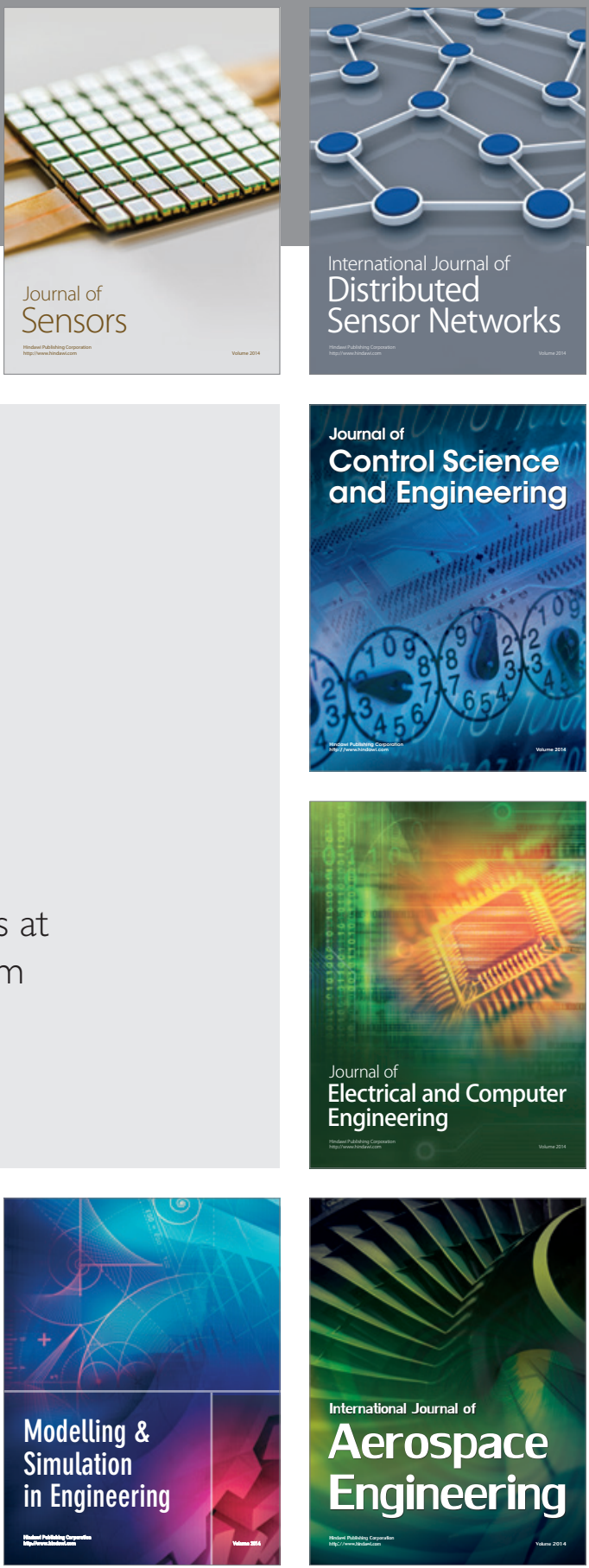

Journal of

Control Science

and Engineering
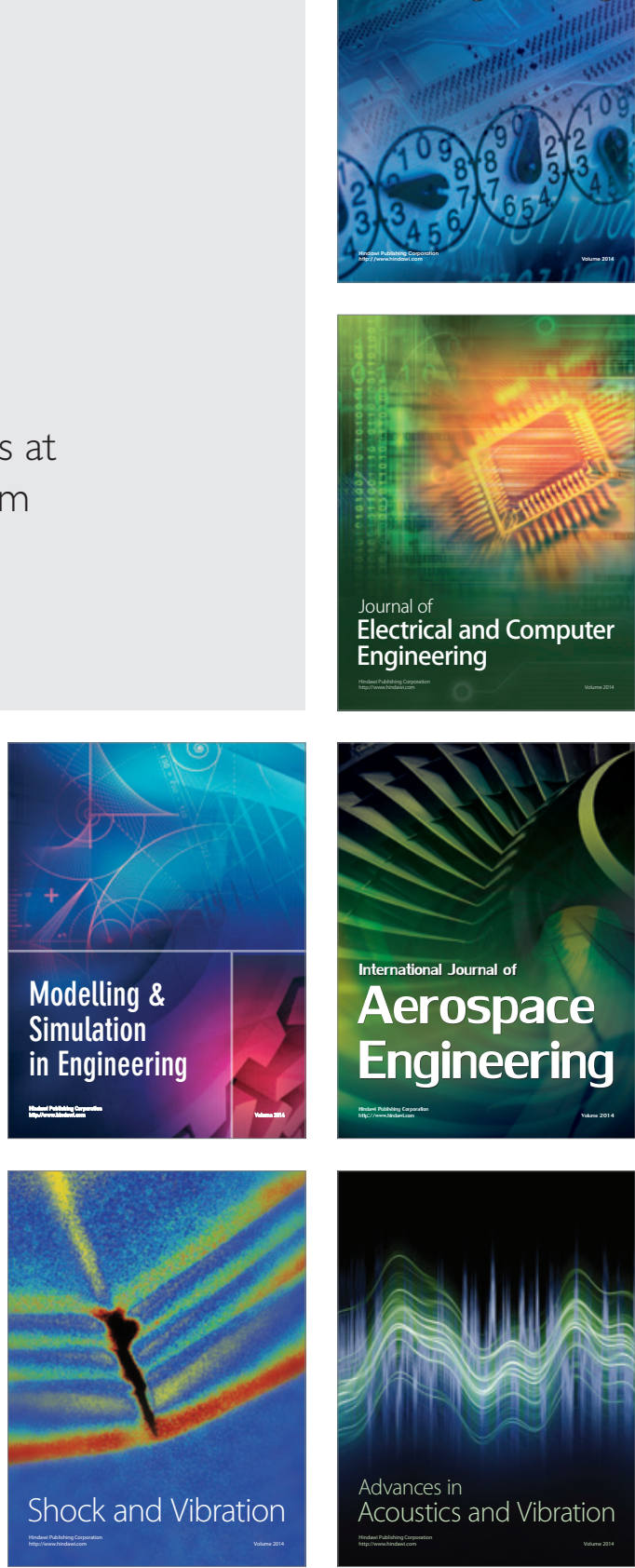